\title{
Po-Г-Ideals in Po-Г-Semigroups
}

\author{
V. B. Subrahmanyeswara Rao Seetamraju ${ }^{1}$, A. Anjaneyulu ${ }^{2}$, \\ D. Madhusudana Rao ${ }^{3}$. \\ ${ }^{I}$ Dept. of Mathematics, $V K R, V N B \& A G K$ College Of Engineering, Gudivada, A.P. India. \\ ${ }^{2,3}$ Dept. of Mathematics, V $R \& N V R$ College, Tenali, A. P. India.
}

\begin{abstract}
In this paper the terms; a completely prime po- $\Gamma$-ideal, $c$-system, a prime po- $\Gamma$-ideal, $m$-system of a po- $\boldsymbol{\Gamma}$-semigroup are introduced. It is proved that every po- $\boldsymbol{\Gamma}$-subsemigroup of a po- $\boldsymbol{\Gamma}$-semigroup is a c-system. It is also proved that a po- $\boldsymbol{\Gamma}$-ideal $P$ of a po- $\boldsymbol{\Gamma}$-semigroup $S$ is completely prime if and only if $S \backslash P$ is either a c-system or empty. It is proved that if $P$ is a po- $\Gamma$-ideal of a po- $\Gamma$-semigroup $S$, then the conditions (1) if $A, B$ are po- $\Gamma$-ideals of $S$ and $A \Gamma B \subseteq P$ then either $A \subseteq P$ or $B \subseteq P$, (2) if $a, b \in S$ such that $a \Gamma S^{l} \Gamma b \subseteq P$, then either $a \in P$ or $b \in P$, are equivalent. It is proved that every completely prime po- $\Gamma$-ideal of a po- $\Gamma$-semigroup $S$ is a prime po- $\boldsymbol{\Gamma}$-ideal of $\boldsymbol{S}$. It is also proved that in a commutative po- $\boldsymbol{\Gamma}$-semigroup $\boldsymbol{S}$, a po- $\boldsymbol{\Gamma}$-ideal $\boldsymbol{P}$ is a prime po- $\boldsymbol{\Gamma}$-ideal if and only if $\boldsymbol{P}$ is a completely prime po- $\boldsymbol{\Gamma}$-ideal. Further it is proved that a po- $\boldsymbol{\Gamma}$-ideal $\boldsymbol{P}$ of a po- $\boldsymbol{\Gamma}$-semigroup $S$ is a prime po- $\Gamma$-ideal of $S$ if and only if $S \backslash P$ is an $m$-system or empty. In a globally idempotent po- $\Gamma$-semigroup, it is proved that every maximal po- $\Gamma$-ideal is a prime po- $\Gamma$-ideal. It is also proved that a globally idempotent po- $\Gamma$-semigroup having a maximal po- $\Gamma$-ideal, contains semisimple elements. The terms completely semiprime po- $\Gamma$-ideal, a semiprime po- $\Gamma$-ideal, $n$-system, $d$-system are introduced. It is proved that (1) every completely semiprime po- $\Gamma$-ideal of a po- $\Gamma$-semigroup is a semiprime po- $\Gamma$-ideal, (2) every completely prime po- $\Gamma$-ideal of a po- $\Gamma$-semigroup is a completely semiprime po- $\Gamma$-ideal. It is also proved that the nonempty intersection of any family of (1) completely prime po- $\Gamma$-ideals of a po- $\Gamma$-semigroup is a completely semiprime po- $\Gamma$-ideal, (2) prime po- $\Gamma$-ideals of a po- $\Gamma$-semigroup is a semiprime po- $\Gamma$-ideal. It is also proved that a po- $\Gamma$-ideal $Q$ of a po- $\Gamma$-semigroup $S$ is a semiprime iff $S \backslash Q$ is either an $n$-system or empty. Further it is proved that if $N$ is an $n$-system in a po- $\Gamma$-semigroup $S$ and $a \in N$, then there exists an $m$-system $M$ of $S$ such that $a \in M$ and $M \subseteq N$. Mathematics Subject Clasification (2010) : 06F05, 06F99, 20M10, $20 M 99$
\end{abstract}

Keywords: A po- $\Gamma$-semigroup, po- $\Gamma$-ideal, prime po- $\Gamma$-ideal, a completely prime po- $\Gamma$-ideal, a completely semiprime po- $\Gamma$-ideal, a semiprime po- $\Gamma$-ideal, po-c-system, po-d-system, po-m-system, po-n-system.

\section{I . Introduction}

$\Gamma$-semigroup was introduced by Sen and Saha [16] as a generalization of semigroup. Anjaneyulu. A [1], [2] and [3] initiated the study of ideals and radicals in semigroups. Many classical notions of semigroups have been extended to $\Gamma$-semigroups by Madhusudhana Rao, Anjaneyulu and Gangadhara Rao [11]. The concept of po-Г-semigroup was introduced by Y. I. Kwon and S. K. Lee [10] in 1996, and it has been studied by several authors. In this paper we introduce the notions of a po- $Г$-semigroups and characterize po- $\Gamma$ semigroups.

\section{II . PRELIMINARIES}

DEFINITION 2.1 : Let $S$ and $\Gamma$ be two non-empty sets. Then $S$ is called a $\Gamma$-semigroup if there exist a mapping from $\mathrm{S} \times \Gamma \times \mathrm{S}$ to $\mathrm{S}$ which maps $(a, \alpha, b) \rightarrow a \alpha b$ satisfying the condition : $(a \gamma b) \mu c=a \gamma(b \mu c)$ for all $a, b, c \in \mathrm{S}$ and $\gamma, \mu \in \Gamma$.

NOTE 2.2 : Let $\mathrm{S}$ be a $\Gamma$-semigroup. If $\mathrm{A}$ and $\mathrm{B}$ are two subsets of $\mathrm{S}$, we shall denote the set $\{a \gamma b: a \in \mathrm{A}$, $b \in \mathrm{B}$ and $\gamma \in \Gamma\}$ by $\mathrm{A} \Gamma \mathrm{B}$.

DEFINITION 2.3: A $\Gamma$-semigroup $S$ is said to a po- $\Gamma$-semigroup if $S$ is a po- set such that $a \leq b \Rightarrow a \gamma c \leq b \gamma c$ and $c \gamma a \leq c \gamma b \forall a, b, c \in S$ and $\gamma \in \Gamma$.

NOTE 2.4: A partially ordered $\Gamma$-semigroup simply called a po- $\Gamma$-semigroup or ordered $\Gamma$-semigroup.

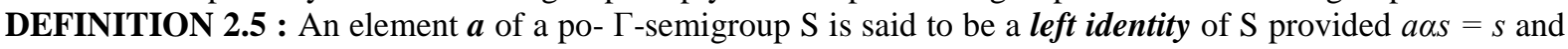
$s \leq a$ for all $s \in S$ and $\alpha \in \Gamma$.

DEFINITION 2.6 : An element $\boldsymbol{a}$ of a po- $\Gamma$-semigroup $\mathrm{S}$ is said to be a right identity of $\mathrm{S}$ provided $s \alpha a=s$ and $s \leq a$ for all $s \in S$ and $\alpha \in \Gamma$.

DEFINITION 2.7 : An element ' $a$ ' of a po- $\Gamma$-semigroup $S$ is said to be a two sided identity or an identity provided it is both a left identity and a right identity of $\mathrm{S}$.

NOTE 2.8 : An element ' $\boldsymbol{a}$ ' of a po- $\Gamma$-semigroup $\mathrm{S}$ is said to be a two sided identity or an identity provided $s \alpha a=a \alpha s=s$ and $s \leq a$ for all $s \in S$ and $\alpha \in \Gamma$. 
THEOREM 2.9 : Any po- $\Gamma$-semigroup $S$ has at most one identity.

NOTE 2.10 : The identity (if exists) of a po- $\Gamma$-semigroup is usually denoted by 1 or $e$.

DEFINITION 2.11 : An element $a$ of a po- $\Gamma$-semigroup $\mathrm{S}$ is said to be a left zero of $\mathrm{S}$ provided $a \alpha s=a$ and $a$ $\leq s$ for all $s \in \mathrm{S}$ and $\alpha \in \Gamma$.

DEFINITION 2.12 : An element $a$ of a po- $\Gamma$-semigroup $\mathrm{S}$ is said to be a right zero of S provided $s \alpha a=a$ and $a$ $\leq s$ for all $s \in \mathrm{S}$ and $\alpha \in \Gamma$.

DEFINITION 2.13 : An element $a$ of a po- $\Gamma$-semigroup $S$ is said to be a two sided zero or zero provided it is both a left zero and a right zero of $\mathrm{S}$.

NOTE 2.14 : An element $a$ of a po- $\Gamma$-semigroup $\mathrm{S}$ is said to be a two sided zero or zero provided $a \alpha s=s \alpha a=a$ and $a \leq s$ for all $s \in \mathrm{S}$ and $\alpha \in \Gamma$.

DEFINITION 2.15 : A po- $\Gamma$-semigroup in which every element is a left zero is called a left zero po- $\Gamma$-semigroup.

DEFINITION 2.16 : A po- $\Gamma$-semigroup in which every element is a right zero is called a right zero po- $\Gamma$-semigroup.

DEFINITION 2.17 : A po- $\Gamma$-semigroup with 0 in which the product of any two elements equals to 0 is called a zero po- $\Gamma$-semigroup or a null po- $\Gamma$-semigroup.

NOTATION 2.18 : Let $\mathrm{S}$ be a po- $\Gamma$-semigroup and $\mathrm{T}$ is a nonempty subset of $\mathrm{S}$. If $\mathrm{H}$ is a nonempty subset of $\mathrm{T}$, we denote the set $\{t \in \mathrm{T}: t \leq h$ for some $h \in \mathrm{H}\}$ by $(\mathrm{H}]_{\mathrm{T}}$. The $\{t \in \mathrm{T}: h \leq t$ for some $h \in \mathrm{H}\}$ by $[\mathrm{H})_{\mathrm{T}}$. Also $(\mathrm{H}]_{\mathrm{s}}$ and $[\mathrm{H})_{\mathrm{s}}$ are simply denoted by $(\mathrm{H}]$ and $[\mathrm{H})$ respectively.

DEFINITION 2.19 : Let $\mathrm{S}$ be a po- $\Gamma$-semigroup. A nonempty subset $\mathrm{T}$ of $\mathrm{S}$ is said to be a po-Г-subsemigroup of $\mathrm{S}$ if $a \gamma b \in \mathrm{T}$, for all $a, b \in \mathrm{T}$ and $\gamma \in \Gamma$ and $t \in \mathrm{T}, s \in \mathrm{S}, s \leq t \Rightarrow s \in \mathrm{T}$.

THEOREM 2.20 : A nonempty subset $\mathrm{T}$ of a po- $\Gamma$-semigroup $\mathrm{S}$ is a po- $\Gamma$-subsemigroup of $\mathrm{S}$ iff (1) $\mathrm{T} \Gamma \mathrm{T} \subseteq \mathrm{T}$, (2) $(\mathrm{T}] \subseteq \mathrm{T}$.

THEOREM 2.21 : Let $S$ be a po- $\Gamma$-semigroup and $A$ is a subset of $S$. Then for all $A, B \subseteq S$ (i) $A \subseteq(A]$,

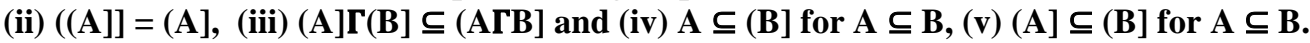

THEOREM 2.22 : The nonempty intersection of two po- $\Gamma$-subsemigroups of a po- $\Gamma$-semigroup $S$ is a po$\Gamma$-subsemigroup of $S$.

THEOREM 2.23 : The nonempty intersection of any family of po- $\Gamma$-subsemigroups of a po- $\Gamma$-semigroup $S$ is a po- $\Gamma$-subsemigroup of $S$.

\section{PO-Г-IDEALS}

We now introduce the term, a left po- $\Gamma$-ideal in a po- $\Gamma$-semigroup.

DEFINITION 3.1 : A nonempty subset A of a po- $\Gamma$-semigroup $\mathrm{S}$ is said to be a left po- $\boldsymbol{\Gamma}$-ideal of $\mathrm{S}$ if

(1) $s \in S, a \in A, \alpha \in \Gamma$ implies $s \alpha a \in A$.

(2) $s \in S, a \in A, s \leq a \Rightarrow s \in \mathrm{A}$.

NOTE 3.2 : A nonempty subset $A$ of a po- $\Gamma$-semigroup $S$ is a left po- $\Gamma$-ideal of $S$ iff (1) $\mathrm{S} \Gamma \mathrm{A} \subseteq \mathrm{A}$, and (2) (A] $\subseteq \mathrm{A}$.

NOTE 3.3 : Let $S$ be a po- $\Gamma$-semigroup. Then the set

$(\mathrm{S} \Gamma \mathrm{a}]=\{t \in \mathrm{S} / t \leq x \alpha a$ for some $x \in \mathrm{S}$ and $\alpha \in \Gamma\}$

THEOREM 3.4: Let $S$ be a po- $\Gamma$-semigroup. Then $(S \Gamma a]$ is a left po- $\Gamma$-ideal of $S$ for all $a \in S$.

Proof : Since $(\mathrm{S} \Gamma \mathrm{a}] \Gamma(\mathrm{S} \Gamma \mathrm{a}] \subseteq(\mathrm{S} \Gamma \mathrm{a} \Gamma \mathrm{S} \Gamma \mathrm{a}]=(\mathrm{S} \Gamma \mathrm{S} \Gamma \mathrm{a}]=(\mathrm{S} \Gamma \mathrm{a}]$.

Therefore ( $\mathrm{S} \Gamma \mathrm{a}]$ is the nonempty subset of $\mathrm{S}$. Let $t \in(\mathrm{S} \Gamma \mathrm{a}], s \in \mathrm{S}, \gamma \in \Gamma$.

$t \in(\mathrm{S} \Gamma \mathrm{a}] \Rightarrow t \leq s_{1} \alpha a$ where $s_{1} \in \mathrm{S}$ and $\alpha \in \Gamma$.

Now $s \gamma t \leq s \mathcal{\gamma}\left(s_{1} \alpha a\right)=\left(s \gamma s_{1}\right) \alpha a \in(\mathrm{S} \Gamma \mathrm{a}]$

Therefore $t \in(\mathrm{S} \Gamma \mathrm{a}], s \in \mathrm{S}, \gamma \in \Gamma \Rightarrow s \gamma t \in(\mathrm{S} \Gamma \mathrm{a}]$ and hence (SГa] is a left po- $\Gamma$-ideal of $\mathrm{S}$.

THEOREM 3.5 : The nonempty intersection of any two left po- $\Gamma$-ideals of a po- $\Gamma$-semigroup $S$ is a left po$\Gamma$-ideal of $\mathrm{S}$.

THEOREM 3.6 : The nonempty intersection of any family of po- left $\Gamma$-ideals of a po- $\Gamma$-semigroup $S$ is a left po-r-ideal of $S$.

THEOREM 3.7 : The union of any two left po- $\Gamma$-ideals of a po- $\Gamma$-semigroup $S$ is a left po- $\Gamma$-ideal of $S$.

THEOREM 3.8 : The union of any family of left po- $\Gamma$-ideals of a po- $\Gamma$-semigroup $S$ is a left po- $\Gamma$-ideal of $\mathrm{S}$.

We now introduce the notion of a right po- $\Gamma$-ideal in a po- $\Gamma$-semigroup.

DEFINITION 3.9 : A nonempty subset A of a po- $\Gamma$-semigroup $\mathrm{S}$ is said to be a right po- $\boldsymbol{\Gamma}$-ideal of $\mathrm{S}$ if

(1) $s \in S, a \in A, \alpha \in \Gamma$ implies $a \alpha s \in A$.

(2) $s \in S, a \in A, s \leq a \Rightarrow s \in \mathrm{A}$.

NOTE 3.10 : A nonempty subset $A$ of a $\Gamma$-semigroup $S$ is a po- right $\Gamma$ - ideal of $S$ iff (1) $\mathrm{A} \Gamma \mathrm{S} \subseteq \mathrm{A}$ and $(2)(\mathrm{A}] \subseteq \mathrm{A}$. 
NOTE 3.11 : Let $S$ be a po- $\Gamma$-semigroup. Then the set (aГS $]=\{t \in \mathrm{S} / t \leq a \alpha x$ for some $x \in \mathrm{S}$ and $\alpha \in \Gamma\}$

THEOREM 3.12: Let $S$ be a po- $\Gamma$-semigroup. Then $(\mathrm{a} \Gamma \mathrm{S}]$ is a po- right $\Gamma$-ideal of $S$ for all $a \in S$.

Proof: Since $(\mathrm{a} \Gamma \mathrm{S}] \Gamma(\mathrm{a} \Gamma \mathrm{S}] \subseteq(\mathrm{a} \Gamma \mathrm{S} \Gamma \mathrm{a} \Gamma \mathrm{S}]=(\mathrm{a} \Gamma \mathrm{a} \Gamma \mathrm{S}]=(\mathrm{a} \Gamma \mathrm{S}]$.

Therefore (a $\Gamma \mathrm{S}]$ is the nonempty subset of S. Let $t \in(\mathrm{a} \Gamma \mathrm{S}], s \in \mathrm{S}, \gamma \in \Gamma$.

$t \in(\mathrm{a} \Gamma \mathrm{S}] \Rightarrow t \leq a \alpha s_{1}$ where $s_{1} \in \mathrm{S}$ and $\alpha \in \Gamma$.

Now $t \gamma s \leq\left(a \alpha s_{1}\right) \gamma s=a \alpha\left(s \gamma s_{1}\right) \in \mathrm{a} \Gamma \mathrm{S} \Rightarrow t \gamma s \in(\mathrm{a} \Gamma \mathrm{S}]$

Therefore $t \in(\mathrm{a} \Gamma \mathrm{S}], s \in \mathrm{S}, \gamma \in \Gamma \Rightarrow t \gamma s \in(\mathrm{a} \Gamma \mathrm{S}]$ and hence $(\mathrm{a} \Gamma \mathrm{S}]$ is a right po- $\Gamma$-ideal of $\mathrm{S}$.

THEOREM 3.13 : The nonempty intersection of any two right po- $\Gamma$-ideals of a po- $\Gamma$-semigroup $S$ is a right po- $\Gamma$-ideal of $S$.

THEOREM 3.14 : The nonempty intersection of any family of right po- $\Gamma$-ideals of a po- $\Gamma$-semigroup $S$ is a right $\Gamma$-ideal of $S$.

THEOREM 3.15 : The union of any two right po- $\Gamma$-ideals of a po- $\Gamma$-semigroup $S$ is a right po- $\Gamma$-ideal of $S$. THEOREM 3.16 : The union of any family of right po- $\Gamma$-ideals of a po- $\Gamma$-semigroup $S$ is a right po- $\Gamma$-ideal of $\mathrm{S}$.

We now introduce the notion of a po- $\Gamma$-ideal of a po- $\Gamma$-semigroup.

DEFINITION 3.17 : A nonempty subset $A$ of a po- $\Gamma$-semigroup $S$ is said to be a two sided po- $\Gamma$ - ideal or simply a po- $\Gamma$ - ideal of $\mathrm{S}$ if

(1) $s \in \mathrm{S}, a \in \mathrm{A}, \alpha \in \Gamma$ imply $s \alpha a \in \mathrm{A}$, a $\alpha s \in \mathrm{A}$.

(2) $s \in S, a \in A, s \leq a \Rightarrow s \in \mathrm{A}$.

NOTE 3.18 : A nonempty subset $A$ of a po- $\Gamma$-semigroup $S$ is a two sided po- $\Gamma$-ideal iff it is both a left po- $\Gamma$-ideal and a right po- $\Gamma$ - ideal of $\mathrm{S}$.

The following examples are due to MANOJ SIRIPITUKDET AND AIYARED IAMPAN [13]

EXAMPLE 3.19 : Let $M=\{a, b, c, d\}$ and $\Gamma=\{\gamma\}$ with the multiplication and the relation $\leq$ on $M$ defined by $x \gamma y=\left\{\begin{array}{l}b \text { if } x, y \in\{a, b\} \\ c \text { otherwise }\end{array}\right.$

and $\leq:=\{(a, a),(b, b),(c, c),(d, d),(b, c),(b, d),(c, d)\}$. Then $\mathrm{M}$ is a po- $\Gamma$-semigroup and $\{b, c\}$ is a po- $\Gamma$-ideal of $\mathrm{M}$.

EXAMPLE 3.20 : Let $\mathrm{S}=\{a, b, c, d\}$ be then a po- $\Gamma$-semigroup defined by the following multiplication and relation $\leq$ on $\mathrm{S}$ as follows:

\begin{tabular}{|c|c|c|c|c|}
\hline$*$ & a & b & c & d \\
\hline a & b & b & d & d \\
\hline b & b & b & d & d \\
\hline c & d & d & c & d \\
\hline d & d & d & d & d \\
\hline
\end{tabular}

$\leq:=\{(a, a),(b, b),(c, c),(d, d),(a, b),(d, b),(d, c)\}$.

Let $\mathrm{M}=\mathrm{S}$ and $\Gamma=\{*\}$. Then $\mathrm{M}$ is a po- $\Gamma$-semigroup and $\{d\}$ is a po- $\Gamma$-ideal of $\mathrm{M}$.

THEOREM 3.21 : Let $S$ be a po- $\Gamma$-semigroup. Then ( $S \Gamma a \Gamma S]$ is a right po- $\Gamma$-ideal of $S$ for all $a \in \mathrm{S}$.

Proof : Since $(\mathrm{S} \Gamma a \Gamma \mathrm{S}] \Gamma(\mathrm{S} \Gamma a \Gamma \mathrm{S}] \subseteq(\mathrm{S} \Gamma a \Gamma \mathrm{S} \Gamma \mathrm{S} \Gamma a \Gamma \mathrm{S}]=(\mathrm{S} \Gamma \mathrm{S} \Gamma a \Gamma \mathrm{S}]=(\mathrm{S} \Gamma a \Gamma \mathrm{S}]$

Therefore (SГa $\Gamma \mathrm{S}]$ is a nonempty subset of $\mathrm{S}$. Let $x \in(\mathrm{S} \Gamma a \Gamma \mathrm{S}], s \in \mathrm{S}$.

$x \in(\mathrm{S} \Gamma a \Gamma \mathrm{S}] \Rightarrow x \leq t \alpha a \beta u$ for some $t, u \in \mathrm{S}$ and $\alpha, \beta \in \Gamma$.

$x \leq t \alpha a \beta u \Rightarrow s \gamma x \leq s \gamma t \alpha a \beta u \Rightarrow s \gamma x \in(\mathrm{S} \Gamma \mathrm{S} \Gamma a \Gamma \mathrm{S}]=(\mathrm{S} \Gamma a \Gamma \mathrm{S}]$

and $x \gamma s \leq t \alpha a \beta u \gamma s \Rightarrow x \gamma s \in(\mathrm{S} \Gamma a \Gamma \mathrm{S} \Gamma \mathrm{S}]=(\mathrm{S} \Gamma a \Gamma \mathrm{S}]$

and $((\mathrm{S} \Gamma a \Gamma \mathrm{S}]] \subseteq(\mathrm{S} \Gamma a \Gamma \mathrm{S}]$ and hence $(\mathrm{S} \Gamma a \Gamma \mathrm{S}]$ is a po- $\Gamma$-ideal of $\mathrm{S}$.

THEOREM 3.22 : The nonempty intersection of any two po- $\Gamma$-ideals of a po- $\Gamma$-semigroup $S$ is a po- $\Gamma$-ideal of $S$.

THEOREM 3.23 : The nonempty intersection of any family of po- $\Gamma$-ideals of a po- $\Gamma$-semigroup $S$ is a po-r-ideal of $S$.

THEOREM 3.24 : The union of any two po- $\Gamma$-ideals of a po- $\Gamma$-semigroup $S$ is a po- $\Gamma$-ideal of $S$. 
THEOREM 3.25 : The union of any family of po- $\Gamma$-ideals of a po- $\Gamma$-semigroup $S$ is a po- $\Gamma$-ideal of $S$.

We now introduce a proper po- $\Gamma$-ideal, trivial po- $\Gamma$-ideal, maximal left po- $\Gamma$-ideal, maximal right po- $\Gamma$-ideal, maximal po- $\Gamma$-ideal and globally idempotent po- $\Gamma$-ideal of a po- $\Gamma$-semigroup.

DEFINITION 3.26 : A po- $\Gamma$-ideal A of a po- $\Gamma$-semigroup $S$ is said to be an proper po- $\Gamma$-ideal of $S$ if $A$ is different from $\mathrm{S}$.

DEFINITION 3.27 : A $\Gamma$-ideal A of a po- $\Gamma$-semigroup $S$ is said to be a trivial po- $\Gamma$-ideal provided $S \backslash A$ is singleton.

DEFINITION 3.28 : A $\Gamma$-ideal A of a po- $\Gamma$-semigroup $S$ is said to be a maximal left po- $\Gamma$-ideal provided A is a proper left po- $\Gamma$-ideal of $\mathrm{S}$ and is not properly contained in any proper left po- $\Gamma$-ideal of $\mathrm{S}$.

DEFINITION 3.29 : A $\Gamma$-ideal A of a po- $\Gamma$-semigroup $S$ is said to be a maximal right po- $\Gamma$-ideal provided $A$ is a proper right $\Gamma$-ideal of $S$ and is not properly contained in any proper right po- $\Gamma$-ideal of $S$.

DEFINITION 3.30 : A $\Gamma$-ideal A of a po- $\Gamma$-semigroup $S$ is said to be a maximal po- $\Gamma$-ideal provided $A$ is a proper $\Gamma$-ideal of $S$ and is not properly contained in any proper po- $\Gamma$-ideal of $S$.

DEFINITION 3.31 : A po- $\Gamma$-ideal A of a po- $\Gamma$-semigroup $\mathrm{S}$ is said to be globally idempotent if $(\mathrm{А} Г А]=A$.

THEOREM 3.32 : If $A$ is a po- $\Gamma$-ideal of a po- $\Gamma$-semigroup $S$ with unity 1 and $1 \in A$ then $A=S$.

Proof: Clearly A $\subseteq$ S. Let $s \in \mathrm{S}$.

$1 \in \mathrm{A}, s \in \mathrm{S}$, A is a po- $\Gamma$-ideal of $\mathrm{S} \Rightarrow 1 \Gamma s \subseteq \mathrm{A}$ and $s \leq 1 \Rightarrow s \in \mathrm{A}$.

Thus $\mathrm{S} \subseteq \mathrm{A}$. $\mathrm{A} \subseteq \mathrm{S}, \mathrm{S} \subseteq \mathrm{A} \Rightarrow \mathrm{S}=\mathrm{A}$.

THEOREM 3.33 : If $S$ is a po- $\Gamma$-semigroup with unity 1 then the union of all proper po- $\Gamma$-ideals of $S$ is the unique maximal po- $\Gamma$-ideal of $S$.

Proof : Let M be the union of all proper po- $\Gamma$-ideals of S. Since 1 is not an element of any proper po- $\Gamma$-ideal of $\mathrm{S}, 1 \notin \mathrm{M}$. Therefore $\mathrm{M}$ is a proper subset of $\mathrm{S}$. By theorem 3.24, $\mathrm{M}$ is a po- $\Gamma$-ideal of $\mathrm{S}$. Thus $\mathrm{M}$ is a proper po- $\Gamma$-ideal of S. Since $M$ contains all proper po- $\Gamma$-ideals of $S, M$ is a maximal po- $\Gamma$-ideal of $S$. If $M_{1}$ is any maximal po- $\Gamma$-ideal of $S$, then $\mathrm{M}_{1} \subseteq \mathrm{M} \subset \mathrm{S}$ and hence $\mathrm{M}_{1}=\mathrm{M}$. Therefore $\mathrm{M}$ is the unique maximal po- $\Gamma$-ideal of $\mathrm{S}$.

We now introducing left po- $\Gamma$-ideal generated by a subset, a right po- $\Gamma$-ideal generated by a subset, po- $\Gamma$-ideal generated by a subset of a po- $\Gamma$-semigroup.

DEFINITION 3.34 : Let $S$ be a po- $\Gamma$-semigroup and $A$ be a nonempty subset of $S$. The smallest po- left $\Gamma$-ideal of $S$ containing $A$ is called left po-r-ideal of $\mathbf{S}$ generated by $\boldsymbol{A}$ and it is denoted by $L(A)$.

THEOREM 3.35 : Let $S$ be a po-r-semigroup and $A$ is a nonempty subset of $S$, then $L(A)=(A \cup S \Gamma A]$.

Proof : Let $s \in \mathrm{S}, r \in(\mathrm{A} \cup \mathrm{S} \Gamma \mathrm{A}]$ and $\gamma \in \Gamma$.

$r \in(\mathrm{A} \cup \mathrm{S} \Gamma \mathrm{A}] \Rightarrow r \in(\mathrm{A}]$ or $r \in(\mathrm{S} \Gamma \mathrm{A}] \Rightarrow r \leq a$ or $r \leq t \alpha a$ for some $a \in \mathrm{A}, t \in \mathrm{S}, \alpha \in \Gamma$.

If $r \leq a$ then $s \gamma r \leq s \gamma a \Rightarrow s \gamma r \in(\mathrm{S} \Gamma \mathrm{A}] \subseteq(\mathrm{A} \cup \mathrm{S} \Gamma \mathrm{A}]$.

If $r \leq t \alpha a$ then $s \gamma r \leq s \gamma(t \alpha a)=(s \gamma t) \alpha a \in \mathrm{S} \Gamma a \Rightarrow s \gamma r \in(\mathrm{S} \Gamma \mathrm{A}] \subseteq(\mathrm{A} \cup \mathrm{S} \Gamma \mathrm{A}]$.

Therefore $s \gamma a \in(\mathrm{A} \cup \mathrm{S} \Gamma \mathrm{A}]$ and hence $(\mathrm{A} \cup \mathrm{S} \Gamma \mathrm{A}]$ is a po- left $\Gamma$-ideal of $\mathrm{S}$.

Let $\mathrm{L}$ be a left po- $\Gamma$-ideal of $\mathrm{S}$ containing A.

Let $r \in(\mathrm{A} \cup \mathrm{S} \Gamma \mathrm{A}]$. Then $r \leq a$ or $r \leq t \alpha a$ for some $a \in \mathrm{A}, t \in \mathrm{S}, \alpha \in \Gamma$.

If $r \leq a$ then $r \leq a \in \mathrm{L}$. If $r \leq t \alpha a$ then $r \leq t \alpha a \in \mathrm{L}$.

Therefore $(\mathrm{A} \cup \mathrm{S} \Gamma \mathrm{A}] \subseteq \mathrm{L}$ and hence $(\mathrm{A} \cup \mathrm{S} \Gamma \mathrm{A}]$ is the smallest left po- $\Gamma$-ideal containing $\mathrm{A}$.

Therefore $\mathrm{L}(\mathrm{A})=(\mathrm{A} \cup \mathrm{S} \Gamma \mathrm{A}]$.

THEOREM 3.36 : The left po- $\Gamma$-ideal of a po- $\Gamma$-semigroup $S$ generated by a nonempty subset $A$ is the intersection of all left po- $\Gamma$-ideals of $S$ containing $A$.

Proof : Let $\Delta$ be the set of all left po- $\Gamma$-ideals of S containing A.

Since $\mathrm{S}$ itself is a left po- $\Gamma$-ideal of $\mathrm{S}$ containing $\mathrm{A}, \mathrm{S} \in \Delta$. So $\Delta \neq \emptyset$.

Let $T^{*}=\bigcap_{T \in \Delta} T$. Since $A \subseteq T$ for all $\mathrm{T} \in \Delta, A \subseteq T^{*}$.

By theorem 3.6, $\mathrm{T}^{*}$ is a left po- $\Gamma$-ideal of $\mathrm{S}$.

Let $\mathrm{K}$ is a left po- $\Gamma$-ideal of $\mathrm{S}$ containing $\mathrm{A}$.

Clearly $\mathrm{A} \subseteq \mathrm{K}$ and $\mathrm{K}$ is a left po- $\Gamma$-ideal of $\mathrm{S}$.

Therefore $K \in \Delta \Rightarrow T^{*} \subseteq K$. Therefore $T^{*}$ is the left po- $\Gamma$-ideal of $S$ generated by $A$.

DEFINITION 3.37 : Let $S$ be a po- $\Gamma$-semigroup and A be a nonempty subset of $S$. The smallest po- right $\Gamma$ ideal of $S$ containing $A$ is called right po- -ideal of $\mathbf{S}$ generated by $\boldsymbol{A}$ and it is denoted by $\mathrm{R}(\mathrm{A})$.

THEOREM 3.38 : Let $S$ be a po- $\Gamma$-semigroup and $A$ is a nonempty subset of $S$, then $R(A)=(A \cup A \Gamma S]$.

Proof : Let $s \in \mathrm{S}, r \in(\mathrm{A} \cup \mathrm{A} \Gamma \mathrm{S}]$ and $\gamma \in \Gamma$.

$r \in(\mathrm{A} \cup \mathrm{A} \Gamma \mathrm{S}] \Rightarrow r \in(\mathrm{A}]$ or $r \in(\mathrm{A} \Gamma \mathrm{S}] \Rightarrow r \leq a$ or $r \leq a a t$ for some $a \in \mathrm{A}, t \in \mathrm{S}, \alpha \in \Gamma$.

If $r \leq a$ then $r \gamma s \leq a \gamma s \Rightarrow r \gamma s \in(\mathrm{A} \Gamma \mathrm{S}] \subseteq(\mathrm{A} \cup \mathrm{A} \Gamma \mathrm{S}]$.

If $r \leq a a t$ then $r \gamma s \leq(a \alpha t) \gamma s=a \alpha(t \gamma s) \in \mathrm{A} \Gamma \mathrm{S} \Rightarrow r \gamma s \in(\mathrm{A} \Gamma \mathrm{S}] \subseteq(\mathrm{A} \cup \mathrm{A} \Gamma \mathrm{S}]$.

Therefore $r / s \in(\mathrm{A} \cup \mathrm{A} \Gamma \mathrm{S}]$ and hence $(\mathrm{A} \cup \mathrm{A} \Gamma \mathrm{S}]$ is a right po- $\Gamma$-ideal of $\mathrm{S}$.

Let $\mathrm{R}$ be a right po- $\Gamma$-ideal of $\mathrm{S}$ containing $\mathrm{A}$. 
Let $r \in$ (A $\cup \mathrm{A} \Gamma \mathrm{S}]$. Then $r \leq a$ or $r \leq a a t$ for some $a \in \mathrm{A}, t \in \mathrm{S}, \alpha \in \Gamma$.

If $r \leq a$ then $r \leq a \in \mathrm{R}$. If $r \leq a a t$ then $r \leq a a t \in \mathrm{R}$.

Therefore $(\mathrm{A} \cup \mathrm{A} \Gamma \mathrm{S}] \subseteq \mathrm{R}$ and hence $(\mathrm{A} \cup \mathrm{A} \Gamma \mathrm{S}]$ is the smallest right po- $\Gamma$-ideal containing $\mathrm{A}$.

Therefore $\mathrm{R}(\mathrm{A})=(\mathrm{A} \cup \mathrm{A} \Gamma \mathrm{S}]$.

THEOREM 3.39 : The right po- $\Gamma$-ideal of a po- $\Gamma$-semigroup $S$ generated by a nonempty subset $A$ is the intersection of all right po- $\Gamma$-ideals of $S$ containing $A$.

Proof : Let $\Delta$ be the set of all right po- $\Gamma$-ideals of $S$ containing A.

Since $\mathrm{S}$ itself is a right po- $\Gamma$-ideal of $\mathrm{S}$ containing $\mathrm{A}, \mathrm{S} \in \Delta$. So $\Delta \neq \emptyset$.

Let $T^{*}=\bigcap_{T \in \Delta} T$. Since $A \subseteq T$ for all $\mathrm{T} \in \Delta, A \subseteq T^{*}$.

By theorem 3.14, $\mathrm{T}^{*}$ is a right po- $\Gamma$-ideal of $\mathrm{S}$.

Let $\mathrm{K}$ is a right po- $\Gamma$-ideal of $\mathrm{S}$ containing $\mathrm{A}$.

Clearly $\mathrm{A} \subseteq \mathrm{K}$ and $\mathrm{K}$ is a right po- $\Gamma$-ideal of $\mathrm{S}$.

Therefore $K \in \Delta \Rightarrow T^{*} \subseteq K$. Therefore $T^{*}$ is the right po- $\Gamma$-ideal of $S$ generated by $A$.

DEFINITION 3.40 : Let $S$ be a po- $\Gamma$-semigroup and $A$ be a nonempty subset of $S$. The smallest po- $\Gamma$-ideal of $\mathrm{S}$ containing $\mathrm{A}$ is called $\boldsymbol{p o}$ - $\Gamma$-ideal of $\mathbf{S}$ generated by $\boldsymbol{A}$ and it is denoted by $\mathrm{J}(\mathrm{A})$.

THEOREM 3.41 : If $S$ is a po-r-semigroup and $A \subseteq S$ then

$\mathbf{J}(A)=(\mathbf{A} \cup \mathbf{A} \Gamma \mathrm{S} \cup \mathrm{S} \Gamma \mathrm{A} \cup \mathrm{S} \Gamma \mathrm{A} \Gamma \mathrm{S}]$.

Proof: Let $s \in \mathrm{S}, r \in(\mathrm{A} \cup \mathrm{A} \Gamma \mathrm{S} \cup \mathrm{S} \Gamma \mathrm{A} \cup \mathrm{S} \Gamma \mathrm{A} \Gamma \mathrm{S}]$ and $\gamma \in \Gamma$.

$r \in(\mathrm{A} \cup \mathrm{A} \Gamma \mathrm{S} \cup \mathrm{S} \Gamma \mathrm{A} \cup \mathrm{S} \Gamma \mathrm{A} \Gamma \mathrm{S}] \Rightarrow r \leq a$ or $r \leq a \alpha t$ or $r \leq t \alpha a$ or $r \leq t \alpha a \beta u$ for some $a \in \mathrm{A} t, u \in \mathrm{S}$ and $\alpha, \beta \in \Gamma$.

If $r \leq a$ then $r \gamma s \leq a \gamma s \in \mathrm{A} \Gamma S \Rightarrow r \gamma s \in(\mathrm{A} \Gamma S]$ and $s \gamma r \leq s \gamma a \in \mathrm{S} \Gamma \mathrm{A} \Rightarrow s \gamma r \in$ (SГA].

If $r \leq a \alpha t$ then $r \gamma s \leq(a \alpha t) \gamma s=a \alpha(t \gamma s) \in \mathrm{A} \Gamma \mathrm{S} \Rightarrow r \gamma s \in(\mathrm{A} \Gamma S]$

and $s \gamma r \leq s \gamma(a \alpha t)=s \gamma a \alpha t \in \mathrm{S} \Gamma \mathrm{A} \Gamma \mathrm{S} \Rightarrow r \gamma s \in(\mathrm{S} \Gamma \mathrm{A} \Gamma \mathrm{S}]$.

If $r \leq t \alpha a$ then $r \gamma s \leq(t \alpha a) \gamma s=t \alpha a \gamma s \in \mathrm{S} \Gamma \mathrm{A} \Gamma \mathrm{S} \Rightarrow r \gamma s \in(\mathrm{S} \Gamma \mathrm{A} \Gamma \mathrm{S}]$

or $s \gamma r \leq s \gamma(t \alpha a)=(s \gamma t) \alpha a \in \mathrm{S} \Gamma \mathrm{A} \Rightarrow s \gamma r \in(\mathrm{S} \Gamma \mathrm{A}]$.

If $r \leq t \alpha a \beta u$ then $r \gamma s \leq(t \alpha a \beta u) \gamma s=t \alpha a \beta(u \gamma s) \in \mathrm{S} \Gamma \mathrm{A} \Gamma \mathrm{S} \Rightarrow r \gamma s \in(\mathrm{S} \Gamma \mathrm{A} \Gamma \mathrm{S}]$

and $s \gamma r \leq s \gamma(t \alpha a \beta u)=(s \gamma t) \alpha a \beta u \in \mathrm{S} \Gamma \mathrm{A} \Gamma \mathrm{S} \Rightarrow s \gamma r \in(\mathrm{S} \Gamma \mathrm{A} \Gamma \mathrm{S}]$.

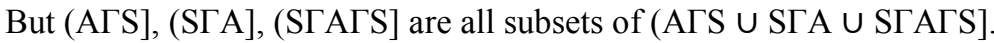

Therefore $r \gamma s, s \gamma r \in(\mathrm{A} \cup \mathrm{A} \Gamma \mathrm{S} \cup \mathrm{S} \Gamma \mathrm{A} \cup \mathrm{S} \Gamma \mathrm{A} \Gamma \mathrm{S}]$ and hence $(\mathrm{A} \cup \mathrm{A} \Gamma \mathrm{S} \cup \mathrm{S} \Gamma \mathrm{A} \cup \mathrm{S} \Gamma \mathrm{A} \Gamma \mathrm{S}]$ is a po- $\Gamma$-ideal of $\mathrm{S}$.

Let $\mathrm{J}$ be a $\Gamma$-ideal of $\mathrm{S}$ containing A. Let $r \in(\mathrm{A} \cup \mathrm{A} \Gamma \mathrm{S} \cup \mathrm{S} \Gamma \mathrm{A} \cup \mathrm{S} \Gamma \mathrm{A} \Gamma \mathrm{S}]$.

Then $r \leq a$ or $r \leq a \alpha t$ or $r \leq t \alpha a$ or $r \leq t \alpha a \beta u$ for some $a \in \mathrm{A} t, u \in \mathrm{S}$ and $\alpha, \beta \in \Gamma$.

If $r \leq a$ then $r \leq a \Rightarrow r \in \mathrm{J}$. If $r \leq a \alpha t$ then $r \leq a \alpha t \Rightarrow r \in \mathrm{J}$.

If $r \leq t \alpha a$ then $r \leq t \alpha a \Rightarrow r \in \mathrm{J}$. If $r \leq t \alpha a \beta u$ then $r \leq t \alpha a \beta u \Rightarrow r \in \mathrm{J}$.

Therefore $(\mathrm{A} \cup \mathrm{A} \Gamma \mathrm{S} \cup \mathrm{S} \Gamma \mathrm{A} \cup \mathrm{S} \Gamma \mathrm{A} \Gamma \mathrm{S}] \subseteq \mathrm{J}$.

Hence (A $\cup \mathrm{A} \Gamma \mathrm{S} \cup \mathrm{S} \Gamma \mathrm{A} \cup \mathrm{S} \Gamma \mathrm{A} \Gamma \mathrm{S}]$ is the smallest po- $\Gamma$-ideal of $\mathrm{S}$ containing $a$.

Therefore $\mathrm{J}(A)=(\mathrm{A} \cup \mathrm{A} \Gamma \mathrm{S} \cup \mathrm{S} \Gamma \mathrm{A} \cup \mathrm{S} \Gamma \mathrm{A} \Gamma \mathrm{S}]$.

THEOREM 3.42 : The po- $\Gamma$-ideal of a $\Gamma$-semigroup $S$ generated by a nonempty subset $A$ is the intersection of all po- $\Gamma$-ideals of $S$ containing $A$.

Proof : Let $\Delta$ be the set of all po- $\Gamma$-ideals of $S$ containing A.

Since $\mathrm{S}$ itself is a po- $\Gamma$-ideal of $\mathrm{S}$ containing $\mathrm{A}, \mathrm{S} \in \Delta$. So $\Delta \neq \emptyset$.

Let $T^{*}=\bigcap_{T \in \Delta} T$. Since $A \subseteq T$ for all $\mathrm{T} \in \Delta, A \subseteq T^{*}$.

By theorem 3.23, $\mathrm{T}^{*}$ is a po- $\Gamma$-ideal of $\mathrm{S}$.

Let $\mathrm{K}$ is a po- $\Gamma$-ideal of $\mathrm{S}$ containing $\mathrm{A}$.

Clearly $\mathrm{A} \subseteq \mathrm{K}$ and $\mathrm{K}$ is a po- $\Gamma$-ideal of $\mathrm{S}$. Therefore $\mathrm{K} \in \Delta \Rightarrow \mathrm{T}^{*} \subseteq \mathrm{K}$.

Therefore $\mathrm{T}^{*}$ is the po- $\Gamma$-ideal of $\mathrm{S}$ generated by A.

po- $\Gamma$-ideal.

We now introduce a principal left po- $Г$-ideal of a po- $Г$-semigroup and characterize principal left

DEFINITION 3.43 : A left po- $Г$-ideal A of a po- $Г$-semigroup $S$ is said to be the principal left po- $\Gamma$-ideal generated by $\boldsymbol{a}$, if A is a po- left $\Gamma$-ideal generated by $\{a\}$ for some $a \in \mathrm{S}$. It is denoted by $\mathrm{L}(a)$.

THEOREM 3.44: If $\mathrm{S}$ is a po- $\Gamma$-semigroup and $a \in \mathrm{S}$ then $\mathrm{L}(a)=(a \cup \mathrm{S} \Gamma a]$.

Proof: $\mathrm{In}$ the theorem 3.35., for $\mathrm{A}=\{a\}$ we have $\mathrm{L}(a)=(a \cup \mathrm{S} \Gamma a]$.

NOTE 3.45: If $\mathrm{S}$ is a po- $\Gamma$-semigroup and $a \in \mathrm{S}$ then $\mathrm{L}(a)=\{t \in \mathrm{S} / t \leq a$ or $t \leq x \gamma a$ for some $x \in \mathrm{S}, \gamma \in \Gamma\}$.

NOTE 3.46 : If $\mathrm{S}$ is a po- $\Gamma$-semigroup and $a \in \mathrm{S}$ then $\mathrm{L}(a)=\left(\mathrm{S}^{1} \Gamma a\right]$. po- $\Gamma$-ideal.

We now introduce principal right po- $Г$-ideal of a po- $Г$-semigroup and characterize principal right

DEFINITION 3.47 : A right po- $Г$-ideal A of a po- $Г$-semigroup $S$ is said to be the principal right po- -ideal generated by $\boldsymbol{a}$ if $\mathrm{A}$ is a right po- $\Gamma$-ideal generated by $\{a\}$ for some $a \in \mathrm{S}$. It is denoted $\mathrm{R}(a)$. 
THEOREM $3.48:$ If $S$ is a po- $\Gamma$-semigroup and $a \in S$ then $R(a)=(a \cup a \Gamma S]$.

Proof: In the theorem 3.38., for $\mathrm{A}=\{a\}$ we have $\mathrm{R}(a)=(a \cup a \Gamma \mathrm{S}]$.

NOTE 3.49: If $\mathrm{S}$ is a po- $\Gamma$-semigroup and $a \in \mathrm{S}$ then $\mathrm{R}(a)=\{t \in \mathrm{S} / t \leq a$ or $t \leq a \gamma x$ for some $x \in \mathrm{S}, \gamma \in \Gamma\}$.

NOTE 3.50 : If $\mathrm{S}$ is a po- $\Gamma$-semigroup and $a \in \mathrm{S}$ then $\mathrm{R}(a)=\left(a \Gamma \mathrm{S}^{1}\right]$.

We now introduce a principal po- $\Gamma$-ideal of a po- $\Gamma$-semigroup and characterize principal po- $\Gamma$-ideal.

DEFINITION 1.3.51 : A po- $\Gamma$-ideal A of a po- $\Gamma$-semigroup $S$ is said to be a principal po- $\Gamma$-ideal provided A is a po- $\Gamma$-ideal generated by $\{a\}$ for some $a \in \mathrm{S}$. It is denoted by $\mathrm{J}[a]$ or $\langle a\rangle$.

THEOREM $3.52:$ If $S$ is a po- $\Gamma$-semigroup and $a \in \mathrm{S}$ then

$\mathrm{J}(a)=(a \cup a \Gamma \mathrm{S} \cup \mathrm{S} \Gamma a \cup \mathrm{S} \Gamma a \Gamma \mathrm{S}]$.

Proof: In the theorem 3.41., for $\mathrm{A}=\{a\}$ we have $\mathrm{J}(a)=(a \cup \mathrm{S} \Gamma a \cup a \Gamma \mathrm{S} \cup \mathrm{S} \Gamma a \Gamma \mathrm{S}]$.

NOTE 3.53:If $\mathrm{S}$ is a po- $\Gamma$-semigroup and $a \in S$, then

$\langle a\rangle=\{t \in \mathrm{S} / t \leq a$ or $t \leq a \gamma x$ or $t \leq x \gamma a$ or $t \leq x \gamma a \delta y$ for some $x, y \in \mathrm{S}$ and $\gamma, \delta \in \Gamma\}$

NOTE 3.54 : If $\mathrm{S}$ is a po- $\Gamma$-semigroup and $a \in S$, then

$\langle a\rangle=(a \cup a \Gamma \mathrm{S} \cup \mathrm{S} \Gamma a \cup \mathrm{S} \Gamma a \Gamma \mathrm{S}]=\left(\mathrm{S}^{1} \Gamma a \Gamma \mathrm{S}^{1}\right]$.

DEFINITION 3.55 : A partial order $\leq$ on a set $\mathrm{S}$ is linear if for any $a, b \in \mathrm{S}$, either $a \leq b$ or $b \leq a$.

DEFINITION 3.56 : Let $\leq$ is a partial order on a set $\mathrm{S}$ is linear. Then $\mathrm{S}$ is called a Chain.

THEOREM 3.57 : In any po-r-semigroup $S$, the following are equivalent.

(1) Principal po- $\Gamma$-ideals of $S$ form a chain.

(2) Po- $\Gamma$-ideals of $S$ form a chain.

Proof : (1) $\Rightarrow(2)$ : Suppose that principal po- $\Gamma$-ideals of $S$ form a chain.

Let A, B be two po- $\Gamma$-ideals of S. Suppose if possible A $\nsubseteq \mathrm{B}, \mathrm{B} \nsubseteq \mathrm{A}$.

Then there exists $a \in \mathrm{A} \backslash \mathrm{B}$ and $\mathrm{b} \in \mathrm{B} \backslash \mathrm{A}$.

$a \in \mathrm{A} \Rightarrow\langle a\rangle \subseteq \mathrm{A}$ and $b \in \mathrm{B} \Rightarrow\langle b\rangle \subseteq \mathrm{B}$.

Since principal po- $\Gamma$-ideals form a chain, either $\langle a\rangle \subseteq\langle b\rangle$ or $\langle b\rangle \subseteq\langle a\rangle$.

If $\langle a\rangle \subseteq\langle b\rangle$, then $a \in\langle b\rangle \subseteq \mathrm{B}$. It is a contradiction.

If $\langle b\rangle \subseteq\langle a\rangle$, then $b \in\langle a\rangle \subseteq \mathrm{A}$. It is also a contradiction.

Therefore either $\mathrm{A} \subseteq \mathrm{B}$ or $\mathrm{B} \subseteq \mathrm{A}$ and hence po- $\Gamma$-ideals from a chain.

(2) $\Rightarrow(1)$ : Suppose that po- $\Gamma$-ideals of $S$ form a chain.

Then clearly principal po- $\Gamma$-ideal of $\mathrm{S}$ form a chain.

We now introduce a left simple po- $\Gamma$-semigroup and characterize left simple po- $\Gamma$-semigroups.

DEFINITION 3.58 : A po- $\Gamma$-semigroup $\mathrm{S}$ is said to be a left simple po- $\boldsymbol{\Gamma}$-semigroup if for every $a, b \in \mathrm{S}$, $\alpha, \beta \in \Gamma$, there exist $x, y \in \mathrm{S}$ such that $b \leq x \alpha a$ and $a \leq y \beta b$.

NOTE 3.59 : A po- $\Gamma$-semigroup $S$ is said to be a left simple po- $\Gamma$-semigroup if $S$ is its only left po- $\Gamma$-ideal.

THEOREM 3.60 : A po- $\Gamma$-semigroup $S$ is a left simple po- $\Gamma$-semigroup if and only if $(\mathrm{S} \Gamma a]=\mathrm{S}$ for all $a \in \mathrm{S}$.

Proof: Suppose that $\mathrm{S}$ is a left simple po- $\Gamma$-semigroup and $a \in \mathrm{S}$.

Let $t \in(\mathrm{S} \Gamma a], s \in \mathrm{S}, \gamma \in \Gamma$.

$t \in(\mathrm{S} \Gamma a] \Rightarrow t \leq s_{1} \alpha a$ where $s_{1} \in S$ and $\alpha \in \Gamma$.

Now $\left.s \gamma t \leq s \mathcal{H} s_{1} \alpha a\right)=\left(s \gamma s_{1}\right) \alpha a \in S \Gamma a \Rightarrow s \gamma t \in(\mathrm{S} \Gamma a]$. Therefore $(\mathrm{S} \Gamma a]$ is a left po- $\Gamma$-ideal of $\mathrm{S}$.

Since $\mathrm{S}$ is a left simple po- $\Gamma$-semigroup, $(\mathrm{S} \Gamma a]=\mathrm{S}$.

Therefore $(\mathrm{S} \Gamma a]=\mathrm{S}$ for all $a \in \mathrm{S}$.

Conversely suppose that $(\mathrm{S} \Gamma a]=\mathrm{S}$ for all $a \in \mathrm{S}$. Let $\mathrm{L}$ be a left $\Gamma$-ideal of $\mathrm{S}$.

Let $l \in \mathrm{L}$. Then $l \in \mathrm{S}$. By assumption, $(\mathrm{S} \Gamma l]=\mathrm{S}$.

Let $s \in \mathrm{S}$. Then $s \in(\mathrm{S} \Gamma l] \Rightarrow s \leq t \alpha l$ for some $t \in \mathrm{S}, \alpha \in \Gamma$.

$l \in \mathrm{L}, t \in \mathrm{S}, \alpha \in \Gamma$ and $\mathrm{L}$ is a left po- $\Gamma$-ideal $\Rightarrow t \alpha l \in \mathrm{L} \Rightarrow s \in \mathrm{L}$. Therefore $\mathrm{S} \subseteq \mathrm{L}$.

Clearly $\mathrm{L} \subseteq \mathrm{S}$ and hence $\mathrm{S}=\mathrm{L}$. Therefore $\mathrm{S}$ is the only left po- $\Gamma$-ideal of $\mathrm{S}$.

Hence $S$ is left simple po- $\Gamma$-semigroup.

We now introduce a right simple po- $\Gamma$-semigroup and characterize right simple po- $\Gamma$-semigroups.

DEFINITION 3.61 : A po- $\Gamma$-semigroup $\mathrm{S}$ is said to be a right simple po- $\Gamma$-semigroup if for every $a, b \in \mathrm{S}$, $\alpha, \beta \in \Gamma$, there exist $x, y \in \mathrm{S}$ such that $b \leq a \alpha x$ and $a \leq b \beta y$.

NOTE 3.62 : A po- $\Gamma$-semigroup $S$ is said to be a right simple po- $\Gamma$-semigroup if $S$ is its only right $\Gamma$-ideal.

THEOREM 3.63: A po- $\Gamma$-semigroup $S$ is a right simple po- $\Gamma$-semigroup if and only if $(a \Gamma S]=S$ for all $a \in S$.

Proof : Suppose that $\mathrm{S}$ is a right simple po- $\Gamma$-semigroup and $a \in \mathrm{S}$.

Let $t \in(a \Gamma \mathrm{S}], s \in \mathrm{S}, \gamma \in \Gamma$.

$t \in(a \Gamma \mathrm{S}] \Rightarrow t \leq a \alpha s_{1}$ where $s_{1} \in S$ and $\alpha \in \Gamma$.

Now $t \gamma s \leq\left(a \alpha s_{1}\right) \gamma s=a \alpha\left(s_{1} \gamma s\right) \in a \Gamma S \Rightarrow t \gamma s \in(a \Gamma \mathrm{S}]$. Therefore $(a \Gamma \mathrm{S}]$ is a right po- $\Gamma$-ideal of S.

Since $\mathrm{S}$ is a right simple po- $\Gamma$-semigroup, $(a \Gamma \mathrm{S}]=\mathrm{S}$. Therefore $(a \Gamma \mathrm{S}]=\mathrm{S}$ for all $a \in \mathrm{S}$. 
Conversely suppose that $(a \Gamma \mathrm{S}]=\mathrm{S}$ for all $a \in \mathrm{S}$.

Let $\mathrm{R}$ be a right po- $\Gamma$-ideal of a po- $\Gamma$-semigroup $\mathrm{S}$.

Let $r \in \mathrm{R}$. Then $r \in \mathrm{S}$. By assumption $(r \Gamma \mathrm{S}]=\mathrm{S}$.

Let $s \in \mathrm{S}$. Then $s \in(r \Gamma \mathrm{S}] \Rightarrow s \leq r \alpha t$ for some $t \in \mathrm{S}, \alpha \in \Gamma$.

$r \in \mathrm{R}, t \in \mathrm{S}, \alpha \in \Gamma$ and $\mathrm{R}$ is a right po- $\Gamma$-ideal $\Rightarrow r \alpha t \in \mathrm{R} \Rightarrow s \in \mathrm{R}$.

Therefore $\mathrm{S} \subseteq \mathrm{R}$. Clearly $\mathrm{R} \subseteq \mathrm{S}$ and hence $\mathrm{S}=\mathrm{R}$.

Therefore $S$ is the only right po- $\Gamma$-ideal of $S$. Hence $S$ is right simple po- $\Gamma$-semigroup.

We now introduce a simple po- $\Gamma$-semigroup and characterize simple po- $\Gamma$-semigroups.

DEFINITION 3.64 : A po- $\Gamma$-semigroup $\mathrm{S}$ is said to be a simple po- $\Gamma$-semigroup if for every $a, b \in \mathrm{S}, \alpha, \beta \in \Gamma$, there exist $x, y \in \mathrm{S}$ such that $a \leq x a b \beta y$.

NOTE 3.65 : A po- $\Gamma$-semigroup $S$ is said to be simple po- $\Gamma$-semigroup if $S$ is its only two-sided po- $\Gamma$-ideal.

THEOREM 3.66 : If $S$ is a left simple po- $\Gamma$-semigroup or a right simple po- $\Gamma$-semigroup then $S$ is a simple po- $\Gamma$-semigroup.

Proof : Suppose that $S$ is a left simple po- $\Gamma$-semigroup. Then $S$ is the only left po- $\Gamma$-ideal of $S$. If $A$ is a po- $\Gamma$-ideal of $S$, then $A$ is a left po- $\Gamma$-ideal of $S$ and hence $A=S$.

Therefore $\mathrm{S}$ itself is the only po- $\Gamma$-ideal of $\mathrm{S}$ and hence $\mathrm{S}$ is a simple po- $\Gamma$-semigroup.

Suppose that $\mathrm{S}$ is a right simple po- $\Gamma$-semigroup. Then $\mathrm{S}$ is the only right po- $\Gamma$-ideal of $\mathrm{S}$. If $\mathrm{A}$ is a po- $\Gamma$-ideal of $\mathrm{S}$, then $\mathrm{A}$ is a right po- $\Gamma$-ideal of $\mathrm{S}$ and hence $\mathrm{A}=\mathrm{S}$.

Therefore $\mathrm{S}$ itself is the only po- $\Gamma$-ideal of $\mathrm{S}$ and hence $\mathrm{S}$ is a simple po- $\Gamma$-semigroup.

THEOREM 3.67 : A po- $\Gamma$-semigroup $S$ is simple po- $\Gamma$-semigroup if and only if $(\mathrm{S} \Gamma \boldsymbol{} \boldsymbol{} \Gamma \mathrm{S}]=\mathrm{S}$ for all $a \in \mathrm{S}$.

Proof: Suppose that $\mathrm{S}$ is a simple po- $\Gamma$-semigroup and $a \in \mathrm{S}$.

Let $t \in(\mathrm{S} \Gamma a \Gamma \mathrm{S}], s \in \mathrm{S}$ and $\gamma \in \Gamma$.

$t \in(\mathrm{S} \Gamma a \Gamma \mathrm{S}] \Rightarrow t \leq s_{1} \alpha a \beta s_{2}$ where $s_{1}, s_{2} \in S$ and $\alpha, \beta \in \Gamma$.

Now $t \gamma s \leq\left(s_{1} \alpha a \beta s_{2}\right) \gamma s=s_{1} \alpha a \beta\left(s_{2} \gamma s\right) \in \mathrm{S} \Gamma a \Gamma \mathrm{S} \Rightarrow t \gamma s \in(\mathrm{S} \Gamma a \Gamma \mathrm{S}]$

and $s \gamma t \leq s \gamma\left(s_{1} \alpha a \beta s_{2}\right)=\left(s \gamma s_{1}\right) \alpha a \beta s_{2} \in \mathrm{S} \Gamma a \Gamma \mathrm{S} \Rightarrow s \gamma t \in(\mathrm{S} \Gamma a \Gamma \mathrm{S}]$.

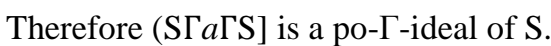

Since $\mathrm{S}$ is a simple po- $\Gamma$-semigroup, $\mathrm{S}$ itself is the only po- $\Gamma$-ideal of $\mathrm{S}$ and hence $(\mathrm{S} \Gamma a \Gamma \mathrm{S}]=\mathrm{S}$.

Conversely suppose that $(\mathrm{S} \Gamma a \Gamma \mathrm{S}]=\mathrm{S}$ for all $a \in \mathrm{S}$. Let I be a $\Gamma$-ideal of $\mathrm{S}$.

Let $a \in \mathrm{I}$. Then $a \in \mathrm{S}$. So $(\mathrm{S} \Gamma a \Gamma \mathrm{S}]=\mathrm{S}$.

Let $s \in \mathrm{S}$. Then $s \in(\mathrm{S} \Gamma a \Gamma \mathrm{S}] \Rightarrow s \leq t_{1} \alpha a \beta t_{2}$ for some $t_{1}, t_{2} \in \mathrm{S}, \alpha, \beta \in \Gamma$.

$a \in \mathrm{I}, t_{1}, t_{2} \in \mathrm{S}, \alpha, \beta \in \Gamma, \mathrm{I}$ is a $\Gamma$-ideal of $\mathrm{S} \Rightarrow t_{1} \alpha a \beta t_{2} \in \mathrm{I} \Rightarrow s \in \mathrm{I}$.

Therefore $\mathrm{S} \subseteq \mathrm{I}$. Clearly $\mathrm{I} \subseteq \mathrm{S}$ and hence $\mathrm{S}=\mathrm{I}$.

Therefore $\mathrm{S}$ is the only $\Gamma$-ideal of $\mathrm{S}$. Hence $\mathrm{S}$ is a simple po- $\Gamma$-semigroup.

We now introduce a regular po- $\Gamma$-ideal of a po- $\Gamma$-semigroup.

DEFINITION 3.68 : A po- $\Gamma$-ideal A of a po- $\Gamma$-semigroup $S$ is said to be regular if every element of $A$ is regular in $\mathrm{A}$.

NOTE 3.69 : A po- $\Gamma$-ideal A of a po- $\Gamma$-semigroup $S$ is said to be regular if $A \subseteq(\mathrm{A} \Gamma \mathrm{S} \Gamma \mathrm{A}]$

THEOREM 3.70 : Every po- $\Gamma$-ideal of a regular po- $\Gamma$-semigroup $S$ is a regular po- $\Gamma$-ideal of $S$.

Proof : Let A be a po- $\Gamma$-ideal of $\mathrm{S}$ and $a \in \mathrm{A}$. Then $a \in \mathrm{S}$ and hence $a$ is regular in $\mathrm{S}$. Therefore $a \leq a a b \beta a$ where $b \in \mathrm{S}$ and $\alpha, \beta \in \Gamma$.

Hence $a \leq a a b \beta a \leq(a a b \beta)(a a b \beta a) \leq a a[(b \beta a) a b] \beta a$.

Let $b_{1}=(b \beta a) \alpha b \in \mathrm{S} \Gamma \mathrm{A} \Gamma \mathrm{S}$. Now $a \leq a \alpha b_{1} \beta a$.

Therefore $a$ is regular in $\mathrm{A}$ and hence $\mathrm{A}$ is a regular po- $\Gamma$-ideal.

\section{Completely Prime Po-Г-Ideals And Prime Po-Г-Ideals}

DEFINITION 4.1 : A po- $\Gamma$-ideal $\mathrm{P}$ of a po- $\Gamma$-semigroup $\mathrm{S}$ is said to be a completely prime po- $\Gamma$ - ideal provided $x, y \in \mathrm{S}$ and $x \Gamma y \subseteq \mathrm{P}$ implies either $x \in \mathrm{P}$ or $y \in \mathrm{P}$.

We now introduce the notion of a $c$-system of a po- $\Gamma$-semigroup.

DEFINITION 4.2 : Let $\mathrm{S}$ be a po- $\Gamma$-semigroup. A nonempty subset $\mathrm{A}$ of $\mathrm{S}$ is said to be a po-c-system of $\mathrm{S}$ if for each $a, b \in \mathrm{A}$ and $\alpha \in \Gamma$ there exists an element $c \in \mathrm{A}$ such that $c \leq a \alpha b$.

NOTE 4.3 : A nonempty subset $\mathrm{A}$ of a po- $\Gamma$-semigroup $\mathrm{S}$ is said to be a po-c-system of $\mathrm{S}$ if for each $a, b \in \mathrm{A}$ there exists an element $c \in \mathrm{A}$ such that $c \in(a \Gamma b]$.

THEOREM 4.4 : Every po- $\Gamma$-subsemigroup of a po- $\Gamma$-semigroup is a po-c-system.

Proof : Let T be a po- $\Gamma$-subsemigroup of $\mathrm{S}$ and $a, b \in \mathrm{T}, \alpha \in \Gamma$.

Since $\mathrm{T}$ is a po- $\Gamma$-subsemigroup of $\mathrm{S}, a a b \in \mathrm{T}$. 
Let $c=a a b \Rightarrow c \leq a a b$. Therefore there exist an element $c \in \mathrm{T}$ such that $c \leq a a b$. Therefore $\mathrm{T}$ is a po-csystem.

THEOREM 4.5: Let $S$ be a po- $\Gamma$-semigroup and $P$ is a po- $\Gamma$-ideal of $S$. Then $(a \Gamma b] \subseteq P$ if and only if $a \Gamma b \subseteq \mathrm{P}$.

Proof : Suppose that $(a \Gamma b] \subseteq \mathrm{P}$. By theorem 2.21, $a \Gamma b \subseteq(a \Gamma b] \subseteq \mathrm{P}$ and hence $a \Gamma b \subseteq \mathrm{P}$. Conversely suppose that $a \Gamma b \subseteq \mathrm{P}$. Let $x \in(a \Gamma b] \Rightarrow x \leq a a b$ where $a a b \in a \Gamma b \Rightarrow x \leq a a b \in a \Gamma b \subseteq \mathrm{P} \Rightarrow x \in \mathrm{P}$. Therefore $(a \Gamma b] \subseteq \mathrm{P}$.

We now prove a necessary and sufficient condition for a po- $\Gamma$-ideal to be a completely prime po- $\Gamma$-ideal in a po- $\Gamma$-semigroup.

THEOREM 4.6 : A po- $\Gamma$-ideal $P$ of a po- $\Gamma$-semigroup $S$ is completely prime if and only if $S \backslash P$ is either a $c$ system of $S$ or empty.

Proof : Suppose that $\mathrm{P}$ is a completely prime po- $\Gamma$-ideal of $\mathrm{S}$ and $\mathrm{S} \backslash \mathrm{P} \neq \varnothing$.

Let $a, b \in \mathrm{S} \backslash \mathrm{P}$. Then $a \notin \mathrm{P}, b \notin \mathrm{P}$. Suppose if possible $c \notin(a \Gamma b]$ for every $c \in \mathrm{S} \backslash \mathrm{P}$.

Then $(a \Gamma b] \subseteq \mathrm{P} \Rightarrow a \Gamma b \subseteq \mathrm{P}$. Since $\mathrm{P}$ is completely prime, either $a \in \mathrm{P}$ or $b \in \mathrm{P}$.

It is a contradiction. Therefore $c \in(a \Gamma b]$ for some $c \in \mathrm{S} \backslash \mathrm{P}$. Hence there exists an element $c \in \mathrm{S} \backslash \mathrm{P}$ such that $c \leq$ $a \alpha b$ for $\alpha \in \Gamma$ and hence S\P is a $c$-system.

Conversely suppose that $\mathrm{S} \backslash \mathrm{P}$ is a $c$-system of $\mathrm{S}$ or $\mathrm{S} \backslash \mathrm{P}$ is empty.

If $\mathrm{S} \backslash \mathrm{P}$ is empty then $\mathrm{P}=\mathrm{S}$ and hence $\mathrm{P}$ is a completely prime.

Assume that $\mathrm{S} \backslash \mathrm{P}$ is a $c$-system of $\mathrm{S}$. Let $a, b \in \mathrm{S}$ and $a \Gamma b \subseteq \mathrm{P}$.

Suppose if possible $a \notin \mathrm{P}$ and $b \notin \mathrm{P}$. Then $a \in \mathrm{S} \backslash \mathrm{P}$ and $b \in \mathrm{S} \backslash \mathrm{P}$.

Since $\mathrm{S} \backslash \mathrm{P}$ is a $c$-system, there exists $c \in \mathrm{S} \backslash \mathrm{P}$ such that $c \leq a \alpha b$ for some $\alpha \in \Gamma$.

$c \leq a a b \in a \Gamma b \subseteq \mathrm{P}$. Thus $c \in \mathrm{P}$.

It is a contradiction. Hence either $a \in \mathrm{P}$ or $b \in \mathrm{P}$.

Therefore $\mathrm{P}$ is a completely prime po- $\Gamma$-ideal of $\mathrm{S}$.

We now introduce the notion of a prime po- $\Gamma$-ideal of a po- $\Gamma$-semigroup.

DEFINITION 4.7 : A po- $\Gamma$-ideal $P$ of a po- $\Gamma$-semigroup $S$ is said to be a prime po- $\Gamma$-ideal provided $A, B$ are two po- $\Gamma$-ideals of $\mathrm{S}$ and $\mathrm{A} \Gamma \mathrm{B} \subseteq \mathrm{P} \Rightarrow$ either $\mathrm{A} \subseteq \mathrm{P}$ or $\mathrm{B} \subseteq \mathrm{P}$.

THEOREM 4.8 : If $P$ is a po- $\Gamma$-ideal of a po- $\Gamma$-semigroup $S$, then the following conditions are equivalent.

(1) If $A, B$ are po- $\Gamma$ - ideals of $S$ and $A \Gamma B \subseteq P$ then either $A \subseteq P$ or $B \subseteq P$.

(2) If $a, b \in \mathrm{S}$ such that $a \Gamma S^{1} \Gamma b \subseteq \mathrm{P}$, then either $a \in \mathrm{P}$ or $b \in \mathrm{P}$.

COROLLARY 4.9 : A po- $\Gamma$-ideal $P$ of a po- $\Gamma$-semigroup $S$ is a prime po- $\Gamma$ - ideal iff $a, b \in S$ such that $a \Gamma S^{1} \Gamma b \subseteq \mathrm{P}$, then either $a \in \mathrm{P}$ or $b \in \mathrm{P}$.

THEOREM 4.10 : Let $P$ be a po- $\Gamma$-ideal of a po- $\Gamma$-semigroup $S$. Then $\left(a \Gamma S^{1} \Gamma b\right] \subseteq P$ if and only if $a \Gamma S^{1} \Gamma b \subseteq \mathrm{P}$.

Proof : Suppose that $\left(a \Gamma \mathrm{S}^{1} \Gamma b\right] \subseteq \mathrm{P}$. By theorem 2.21, $a \Gamma \mathrm{S}^{1} \Gamma b \subseteq\left(a \Gamma \mathrm{S}^{1} \Gamma b\right] \subseteq \mathrm{P}$ and hence $a \Gamma \mathrm{S}^{1} \Gamma b \subseteq \mathrm{P}$.

Conversely suppose that $a \Gamma \mathrm{S}^{1} \Gamma b \subseteq \mathrm{P}$. Let $x \in\left(a \Gamma \mathrm{S}^{1} \Gamma b\right]$

$\Rightarrow x \leq a \alpha s \beta b$ for some $a \alpha s \beta b \in a \Gamma \mathrm{S}^{1} \Gamma b \Rightarrow x \leq a \alpha s \beta b \in a \Gamma \mathrm{S}^{1} \Gamma b \subseteq \mathrm{P} \Rightarrow x \in \mathrm{P}$.

Therefore $\left(a \Gamma \mathrm{S}^{1} \Gamma b\right] \subseteq \mathrm{P}$.

DEFINITION 4.11 : A po- $\Gamma$-ideal A is said to be exceptional prime po- $\Gamma$-ideal if A is a po-prime $\Gamma$-ideal which is not completely prime po- $\Gamma$-ideal.

THEOREM 4.12 : Every completely prime po- $\Gamma$-ideal of a po- $\Gamma$-semigroup $S$ is a prime po- $\Gamma$-ideal of $S$.

Proof: Suppose that A is a completely prime po- $\Gamma$-ideal of a po- $\Gamma$-semigroup S. Let $a, b \in \mathrm{S}$ and $a \Gamma \mathrm{S}{ }^{1} \Gamma \mathrm{b} \subseteq \mathrm{A}$. Now $a \Gamma b \subseteq a \Gamma S^{1} \Gamma b \subseteq \mathrm{A}$.

Since $\mathrm{A}$ is a completely prime, either $a \in \mathrm{A}$ or $b \in \mathrm{A}$.

Therefore $\mathrm{A}$ is a prime po- $\boldsymbol{\Gamma}$-ideal of $\mathrm{S}$.

THEOREM 4.13 : Let $S$ be a commutative po- $\Gamma$-semigroup. A po- $\Gamma$-ideal $P$ of $S$ is a prime po- $\Gamma$-ideal if and only if $P$ is a completely prime po- $\Gamma$-ideal.

Proof: Suppose that $\mathrm{P}$ is a prime po- $\Gamma$-ideal of po- $Г$-semigroup $\mathrm{S}$.

Let $x, y \in \mathrm{S}$ and $x \Gamma y \subseteq \mathrm{P}$. Now $x \Gamma y \subseteq \mathrm{P}, \mathrm{P}$ is a po- $\Gamma$-ideal $\Rightarrow x \Gamma y \Gamma \mathrm{S}^{1} \subseteq \mathrm{P}$.

Since $\mathrm{S}$ is commutative, $x \Gamma \mathrm{S}^{1} \Gamma \mathrm{y}=x \Gamma y \Gamma \mathrm{S}^{1} \subseteq \mathrm{P}$.

By corollary 4.18, either $x \in \mathrm{P}$ or $y \in \mathrm{P}$. Hence $\mathrm{P}$ is a completely prime po- $\Gamma$-ideal.

Conversely suppose that $\mathrm{P}$ is a completely prime po- $\Gamma$-ideal of $\mathrm{S}$.

By theorem 4.19, $\mathrm{P}$ is a prime po- $\Gamma$-ideal of $\mathrm{S}$.

We now introduce the notion of an $m$-system of a po- $\Gamma$-semigroup.

DEFINITION 4.14 : A nonempty subset $A$ of a po- $\Gamma$-semigroup $S$ is said to be an po-m-system provided for any $a, b \in \mathrm{A}$ and $\alpha, \beta \in \Gamma$ there exists an $c \in \mathrm{A}$ and $x \in \mathrm{S}$ such that $c \leq a \alpha x \beta b$.

NOTE 4.15 : A nonempty subset A of a po- $\Gamma$-semigroup $S$ is said to be an $\boldsymbol{m}$-system provided for any $a, b \in \mathrm{A}$ there exists an $c \in \mathrm{A}$ and $x \in \mathrm{S}$ such that $c \in(a \Gamma \mathrm{S} \Gamma b]$. 
THEOREM 4.16 : A nonempty set $A$ is an $m$-system of $\Gamma$-semigroup $(S, \Gamma,$.$) if and only if A$ is an $m$-system of po- $\Gamma$-semigroup $(\mathrm{S}, \boldsymbol{\Gamma}, ., \leq)$.

Proof : Suppose that a nonempty set $A$ is an $m$-system of $\Gamma$-semigroup $S$. Then for each $a, b \in \mathrm{A}$ and $\alpha, \beta \in \Gamma$ there exist an $x \in \mathrm{S}$ such that $a \alpha x \beta b \in \mathrm{A} . \quad a a x \beta b \in \mathrm{A}$. Let $c=a a x \beta b$. Then $\Rightarrow c \leq a \alpha x \beta b$ for $a \alpha x \beta b \in \mathrm{A} \Rightarrow c \in \mathrm{A}$ and hence there exists an element $c \in \mathrm{A}$ such that $c \leq a \alpha x \beta b$. Therefore A is an $m$-system of S.

Conversely suppose that $\mathrm{A}$ is a po- $m$-system of a po- $\Gamma$-semigroup $\mathrm{S}$. Then for each $a, b \in \mathrm{A}$ and $\alpha, \beta \in \Gamma$ there exists an element $c \in \mathrm{A}$ and $x \in \mathrm{S}$ such that $c \leq a \alpha x \beta b$.

$c \leq a \alpha x \beta b \Rightarrow c \leq a \alpha x \beta b \in a \Gamma \mathrm{S} \Gamma b \subseteq \mathrm{A} \Rightarrow a \alpha x \beta b \in \mathrm{A}$ and hence $\mathrm{A}$ is an $m$-system of $\Gamma$-semigroup $\mathrm{S}$.

We now prove a necessary and sufficient condition for a po- $\Gamma$-ideal to be a prime po- $\Gamma$-ideal in a po- $\Gamma$-semigroup.

THEOREM 4.17 : A po- $\Gamma$-ideal $P$ of a po- $\Gamma$-semigroup $S$ is a prime po- $\Gamma$-ideal of $S$ if and only if $S \backslash P$ is an $m$-system of $S$ or empty.

Proof : Suppose that $\mathrm{P}$ is a prime po- $\Gamma$-ideal of a po- $\Gamma$-semigroup $\mathrm{S}$ and $\mathrm{S} \backslash \mathrm{P} \neq \emptyset$.

Let $a, b \in \mathrm{S} \backslash \mathrm{P}$. Then $a \notin \mathrm{P}, b \notin \mathrm{P}$.

Suppose if possible $c \notin\left(a \Gamma S^{1} \Gamma b\right]$ for every $c \in S \backslash P$.

Then $\left(a \Gamma S^{1} \Gamma b\right] \subseteq \mathrm{P} \Rightarrow a \Gamma S^{1} \Gamma b \subseteq \mathrm{P}$. Since $\mathrm{P}$ is prime, either $a \in \mathrm{P}$ or $b \in \mathrm{P}$. It is a contradiction.

Therefore there exist an element $c \in\left(a \Gamma S^{1} \Gamma b\right]$ for some $c \in \mathrm{S} \backslash \mathrm{P}$. Hence there exists $c \in \mathrm{S} \backslash \mathrm{P}$ such that $c \leq a \alpha x \beta b$ for some $a \alpha x \beta b \in a \Gamma S^{1} \Gamma b$. Hence S\P is an $m$-system.

Conversely suppose that $\mathrm{S} \backslash \mathrm{P}$ is either an $m$-system of $\mathrm{S}$ or $\mathrm{S} \backslash \mathrm{P}=\emptyset$.

If $S \backslash P$ is empty then $\mathrm{P}=\mathrm{S}$ and hence $\mathrm{P}$ is a prime po- $\Gamma$-ideal.

Assume that $\mathrm{S} \backslash \mathrm{P}$ is an $m$-system of $\mathrm{S}$.

Let $a, b \in \mathrm{S}$ and $a \Gamma \mathrm{S}^{1} \Gamma b \subseteq \mathrm{P}$. Suppose if possible $a \notin \mathrm{P}, b \notin \mathrm{P}$. Then $a, b \in \mathrm{S} \backslash \mathrm{P}$.

Since $\mathrm{S} \backslash \mathrm{P}$ is an $m$-system, there exists $c \in \mathrm{S} \backslash \mathrm{P}$ such that $c \leq a \alpha x \beta b$ for $x \in \mathrm{S}, \alpha, \beta \in \Gamma$.

$c \leq a \alpha x \beta b \in a \Gamma \mathrm{S}^{1} \Gamma b \subseteq \mathrm{P}$. Thus $c \in \mathrm{P}$.

It is a contradiction. Therefore either $a \in \mathrm{P}$ or $b \in \mathrm{P}$.

Hence $\mathrm{P}$ is a prime po- $\Gamma$-ideal of $\mathrm{S}$.

We now introduce the notion of a globally idempotent po- $\Gamma$-semigroup.

DEFINITION 4.18 : A po- $\Gamma$-semigroup $S$ is said to be a globally idempotent po- $\Gamma$-semigroup if $(\mathrm{S} \Gamma \mathrm{S}]=\mathrm{S}$.

THEOREM 4.19 : If $S$ is a globally idempotent po- $\Gamma$-semigroup then every maximal po- $\Gamma$-ideal of $S$ is a prime po-r-ideal of $S$.

Proof: Let M be a maximal po- $\Gamma$-ideal of S.

Let $\mathrm{A}, \mathrm{B}$ be two po- $\Gamma$-ideals of $\mathrm{S}$ such that $\mathrm{A} \Gamma \mathrm{B} \subseteq \mathrm{M}$.

Suppose if possible $\mathrm{A} \nsubseteq \mathrm{M}, \mathrm{B} \nsubseteq \mathrm{M}$.

Now $\mathrm{A} \nsubseteq \mathrm{M} \Rightarrow \mathrm{M} \cup \mathrm{A}$ is a po- $\Gamma$-ideal of $\mathrm{S}$ and $\mathrm{M} \subset \mathrm{M} \cup \mathrm{A} \subseteq \mathrm{S}$.

Since $M$ is maximal, $M \cup A=S$. Similarly $B \nsubseteq M \Rightarrow M \cup B=S$.

Now $\mathrm{S}=(\mathrm{S} \Gamma \mathrm{S}]=((\mathrm{M} \cup \mathrm{A}) \Gamma(\mathrm{M} \cup \mathrm{B})]=((\mathrm{M} \Gamma \mathrm{M}) \cup(\mathrm{M} \Gamma \mathrm{B}) \cup(\mathrm{A} \Gamma \mathrm{M}) \cup(\mathrm{A} \Gamma \mathrm{B})] \subseteq(\mathrm{M}] \Rightarrow \mathrm{S} \subseteq \mathrm{M}$. Thus $\mathrm{M}=\mathrm{S}$.

It is a contradiction. Therefore either $\mathrm{A} \subseteq \mathrm{M}$ or $\mathrm{B} \subseteq \mathrm{M}$. Hence $\mathrm{M}$ is a prime.

THEOREM 4.20 : If $S$ is a globally idempotent po- $\Gamma$-semigroup having maximal po- $\Gamma$-ideals then $S$ contains semisimple elements.

Proof : Suppose that $\mathrm{S}$ is a globally idempotent po- $\Gamma$-semigroup having maximal po- $\Gamma$-ideals. Let $\mathrm{M}$ be a maximal po- $\Gamma$-ideal of $\mathrm{S}$. Then by theorem $4.30, \mathrm{M}$ is a prime po- $\Gamma$-ideal of $\mathrm{S}$.

Now if $a \in \mathrm{SLM}$ then $\langle a>\Gamma<a>\nsubseteq \mathrm{M} \Rightarrow(\langle a>\Gamma<a>] \nsubseteq \mathrm{M}$

and hence $\mathrm{S}=\mathrm{M} \cup(\langle a\rangle]=\mathrm{M} \cup(<a>\Gamma<a\rangle]$. Therefore $a \in(\langle a\rangle \Gamma<a\rangle]$.

Thus $a$ is semisimple. Therefore $\mathrm{S}$ contains semisimple elements.

\section{V . Completely Semiprime Po-Г-Ideals And Semiprime Po-Г-Ideals}

We now introduce the notion of a completely semiprime po- $\Gamma$-ideal and a semiprime po- $\Gamma$-ideal in a po- $\Gamma$-semigroup.

DEFINITION 5.1 : A po- $Г$-ideal A of a po- $\Gamma$-semigroup $S$ is said to be a completely semiprime po- $\Gamma$ - ideal provided $x \Gamma x \subseteq A ; x \in \mathrm{S}$ implies $x \in \mathrm{A}$.

THEOREM 5.2 : Every completely prime po- $\Gamma$-ideal of a po- $\Gamma$-semigroup $S$ is a completely semiprime po-r-ideal of $S$.

Proof: Let A be a po- completely prime $\Gamma$-ideal of a po- $\Gamma$-semigroup $S$.

Suppose that $x \in S$ and $x \Gamma x \subseteq A$. Since A is a completely prime po- $\Gamma$-ideal of $\mathrm{S}, x \in \mathrm{A}$.

Therefore $S$ is a completely semiprime po- $\Gamma$-ideal.

THEOREM 5.3 : The nonempty intersection of any family of a completely prime po- $\Gamma$-ideals of a po- $\Gamma$ semigroup $S$ is a completely semiprime po- $\Gamma$-ideal of $S$. 
Proof : Let $\left\{\mathrm{A}_{\alpha}\right\}_{\alpha \in \Delta}$ be a family of a completely prime po- $\Gamma$-ideals of $\mathrm{S}$ such that $\bigcap_{\alpha \in \Delta} A_{\alpha} \neq \emptyset$. By theorem 3.26, $\bigcap_{\alpha \in \Delta} A_{\alpha}$ is a po- $\Gamma$-ideal.

Let $a \in \mathrm{S}, a \Gamma a \subseteq \bigcap_{\alpha \in \Delta} A_{\alpha}$. Then $a \Gamma a \subseteq \mathrm{A}_{\alpha}$ for all $\alpha \in \Delta$.

Since $\mathrm{A}_{\alpha}$ is a completely prime, $a \in \mathrm{A}_{\alpha}$ for all $\alpha \in \Delta$ and hence $a \in \bigcap_{\alpha \in \Delta} A_{\alpha}$.

Therefore $\bigcap_{\alpha \in \Delta} A_{\alpha}$ is a completely semiprime po- $\Gamma$-ideal of $\mathrm{S}$.

We now introduce the notion of a $d$-system of a po- $\Gamma$-semigroup.

DEFINITION 5.4 : Let $S$ be a po- $\Gamma$-semigroup. A nonempty subset $A$ of $S$ is said to be a po-d-system of $S$ if for each $a \in \mathrm{A}$ and $\alpha \in \Gamma$, there exists an element $c \in \mathrm{A}$ such that $c \leq a \alpha a$.

NOTE 5.5 : A nonempty subset A of a po- $\Gamma$-semigroup $\mathrm{S}$ is said to be a po- $d$-system of $\mathrm{S}$ if for each $a \in \mathrm{A}$, there exists $c \in$ A such that $c \in(a \Gamma a]$.

We now prove a necessary and sufficient condition for a po- $\Gamma$-ideal to be a completely semiprime po- $\Gamma$-ideal in a po- $\Gamma$-semigroup.

THEOREM 5.6 : A po- $\Gamma$-ideal $P$ of a po- $\Gamma$-semigroup $S$ is a completely semiprime iff $S \backslash P$ is a po- $d$-system of $S$ or empty.

Proof: Suppose that $\mathrm{P}$ is a completely semiprime po- $\Gamma$-ideal of $\mathrm{S}$ and $\mathrm{S} \backslash \mathrm{P} \neq \varnothing$.

Let $a \in \mathrm{S} \backslash \mathrm{P}$. Then $a \notin \mathrm{P}$. Suppose if possible $c \notin(a \Gamma a]$ for every $c \in \mathrm{S} \backslash \mathrm{P}$.

Then $(a \Gamma a] \subseteq \mathrm{P} \Rightarrow a \Gamma a \subseteq \mathrm{P}$. Since $\mathrm{P}$ is a completely semiprime, $a \in \mathrm{P}$.

It is a contradiction. Therefore there exists an element $c \in \mathrm{S} \backslash \mathrm{P}$ such that $c \leq a \alpha a$.

Therefore S\P is a po- $d$-system of S.

Conversely suppose that S\P is a $d$-system of S or S\P is empty.

If $\mathrm{S} \backslash \mathrm{P}$ is empty then $\mathrm{P}=\mathrm{S}$ and hence $\mathrm{P}$ is completely semiprime.

Assume that $\mathrm{S} \backslash \mathrm{P}$ is a po- $d$-system of $\mathrm{S}$.

Let $a \in \mathrm{S}$ and $a \Gamma a \subseteq \mathrm{P}$. Suppose if possible $a \notin \mathrm{P}$. Then $a \in \mathrm{S} \mathrm{P}$.

Since S\P is a $d$-system, there exists an element $c \in \mathrm{S} \backslash \mathrm{P}$ such that $c \leq a \alpha a$ for $\alpha \in \Gamma$.

$c \leq a \alpha a \in a \Gamma a \subseteq \mathrm{P}$. Therefore $c \in \mathrm{P}$. It is a contradiction. Hence $a \in \mathrm{P}$.

Thus $\mathrm{P}$ is a completely semiprime po- $\Gamma$-ideal of $\mathrm{S}$.

We now introduce the notion of a semiprime po- $\Gamma$-ideal of a po- $\Gamma$-semigroup.

DEFINITION 5.7 : A po- $Г$ - ideal A of a po- $Г$-semigroup $S$ is said to be a semiprime po- $\Gamma$-ideal provided $x \in \mathrm{S}, x \Gamma \mathrm{S}^{1} \Gamma x \subseteq A$ implies $x \in \mathrm{A}$.

THEOREM 5.8 : Every completely semiprime po- $\Gamma$-ideal of a po- $\Gamma$-semigroup $S$ is a semiprime po- $\Gamma$-ideal of $\mathrm{S}$.

Proof: Suppose that A is a completely semiprime po- $\Gamma$-ideal of a po- $\Gamma$-semigroup $\mathrm{S}$.

Let $a \in \mathrm{S}$ and $a \Gamma \mathrm{S}^{1} \Gamma a \subseteq \mathrm{A}$.

Now $a \Gamma a \subseteq a \Gamma \mathrm{S}^{1} \Gamma a \subseteq \mathrm{A}$. Since A is a completely semiprime, $a \in \mathrm{A}$.

Therefore A is a semiprime po- $\Gamma$-ideal of $\mathrm{S}$.

THEOREM 5.9 : Let $S$ be a commutative po- $\Gamma$-semigroup. A po- $\Gamma$-ideal A of $S$ is completely semiprime iff it is semiprime.

Proof :Suppose that A is a completely semiprime po- $\Gamma$-ideal of S.

By theorem 5.8, A is a semiprime po- $\Gamma$-ideal of $\mathrm{S}$.

Conversely suppose that $\mathrm{A}$ is a semiprime po- $\Gamma$-ideal of S. Let $x \in \mathrm{S}$ and $x \Gamma x \subseteq \mathrm{A}$.

Now $x \Gamma x \subseteq \mathrm{A} \Rightarrow s \Gamma x \Gamma x \subseteq \mathrm{A}$ for all $s \in \mathrm{S} \Rightarrow x \Gamma s \Gamma x \subseteq \mathrm{A}$ for all $s \in \mathrm{S} \Rightarrow x \Gamma \mathrm{S} \Gamma x \subseteq \mathrm{A}$

$\Rightarrow x \in \mathrm{A}$, since $\mathrm{A}$ is a semiprime.

Therefore A is a completely semiprime po- $\Gamma$-ideal of $S$.

THEOREM 5.10 : Every prime po- $\Gamma$-ideal of a po- $\Gamma$-semigroup $S$ is a semiprime po- $\Gamma$-ideal of $S$.

Proof: Suppose that A is a prime po- $\Gamma$-ideal of a po- $\Gamma$-semigroup $S$.

Let $a \in \mathrm{S}$ and $a \Gamma \mathrm{S}^{1} \Gamma a \subseteq A$. By corollary 4.19, $a \in \mathrm{A}$.

Therefore $\mathrm{A}$ is a semiprime po- $\Gamma$-ideal of $\mathrm{S}$.

THEOREM 5.11 : The nonempty intersection of any family of prime po- $\Gamma$-ideals of a po- $\Gamma$-semigroup $S$ is a semiprime po- $\Gamma$-ideal of $S$.

Proof : Let $\left\{\mathrm{A}_{\alpha}\right\}_{\alpha \in \Delta}$ be a family of a prime po- $\Gamma$-ideals of S such that $\bigcap_{\alpha \in \Delta} A_{\alpha} \neq \emptyset$.

By theorem 3.26, $\bigcap_{\alpha \in \Delta} A_{\alpha}$ is a po- $\Gamma$-ideal. 
Let $a \in \mathrm{S}, a \Gamma \mathrm{S} \Gamma a \subseteq \bigcap_{\alpha \in \Delta} A_{\alpha}$. Then $a \Gamma \mathrm{S} \Gamma a \subseteq \mathrm{A}_{\alpha}$ for all $\alpha \in \Delta$.

Since $\mathrm{A}_{\alpha}$ is prime, $a \in \mathrm{A}_{\alpha}$ for all $\alpha \in \Delta$ and hence $a \in \bigcap_{\alpha \in \Delta} A_{\alpha}$. Therefore $\bigcap_{\alpha \in \Delta} A_{\alpha}$ is a semiprime po- $\Gamma$-ideal of S.

We now introduce the notion of an $n$-system of a po- $\Gamma$-semigroup.

DEFINITION 5.12 : A nonempty subset $A$ of a po- $\Gamma$-semigroup $S$ is said to be an po-n-system provided for any $a \in \mathrm{A}$ and some $\alpha, \beta \in \Gamma$ there exists an element $c \in \mathrm{A}, x \in \mathrm{S}$ such that $c \leq a \alpha x \beta a$.

NOTE 5.13 : A nonempty subset A of a po- $\Gamma$-semigroup $S$ is said to be an po-n-system provided for any $a \in A$, $x \in \mathrm{S}$ there exists an element $c \in \mathrm{A}$ such that $c \in(a \Gamma \mathrm{S} \Gamma a]$.

THEOREM 5.14 : Every po-m-system in a po-r-semigroup $S$ is an po-n-system.

Proof : Let A be po-m-system of a po- $\Gamma$-semigroup S. Let $a \in \mathrm{A}$. Since A is a po-m-system. $a, a \in \mathrm{A}$ and $\alpha, \beta \in \Gamma$ there exists an $c \in \mathrm{A}$ and $x \in \mathrm{S}$ such that $c \leq a \alpha x \beta b \Rightarrow c \leq a \alpha x \beta a$ and hence $\mathrm{A}$ is an po- $n$-system of $\mathrm{S}$.

THEOREM 5.15 : A nonempty set $A$ is an $n$-system of $\Gamma$-semigroup $(S, \Gamma,$.$) if and only if A$ is an $n$-system of a po- $\Gamma$-semigroup $(S, \Gamma, ., \leq)$.

We now prove a necessary and sufficient condition for a po- $\Gamma$-ideal to be a semiprime po- $\Gamma$-ideal in a po-Г-semigroup.

THEOREM 5.16 : A po- $\Gamma$ - ideal $Q$ of a po- $\Gamma$-semigroup $S$ is a semiprime po- $\Gamma$-ideal iff $S \backslash Q$ is a po- $n$-system of $S$ or empty.

Proof : Suppose that $\mathrm{Q}$ is a semiprime po- $\Gamma$-ideal of a po- $\Gamma$-semigroup $S$ and $S \backslash Q \neq \varnothing$.

Let $a \in \mathrm{S} \backslash \mathrm{Q}$. Then $a \notin \mathrm{Q}$. Suppose if possible $c \notin\left(a \Gamma \mathrm{S}^{1} \Gamma a\right]$ for every $c \in \mathrm{S} \backslash \mathrm{Q}$.

Then $\left(a \Gamma S^{1} \Gamma a\right] \subseteq \mathrm{Q} \Rightarrow a \Gamma \mathrm{S}^{1} \Gamma a \subseteq \mathrm{Q}$. Since $\mathrm{Q}$ is a semiprime, $a \in \mathrm{Q}$. It is a contradiction.

Therefore there exist an element $c \in \mathrm{S} \backslash \mathrm{Q}$ such that $c \leq a \alpha x \beta a$ for some $a \alpha x \beta a \in a \Gamma \mathrm{S}^{1} \Gamma a$.

Hence S\Q is an $n$-system.

Conversely suppose that $\mathrm{S} \backslash \mathrm{Q}$ is either an $n$-system of $\mathrm{S}$ or $\mathrm{S} \backslash \mathrm{Q}=\emptyset$.

If $\mathrm{S} \backslash \mathrm{Q}$ is empty then $\mathrm{Q}=\mathrm{S}$ and hence $\mathrm{Q}$ is a semiprime.

Assume that $\mathrm{S} \backslash \mathrm{Q}$ is an $n$-system of $\mathrm{S}$. Let $a \in \mathrm{S}$ and $a \Gamma \mathrm{S}{ }^{1} \Gamma a \subseteq \mathrm{Q}$.

Suppose if possible $a \notin \mathrm{Q}$. Then $a \in \mathrm{S} \backslash \mathrm{Q}$. Since $\mathrm{S} \backslash \mathrm{Q}$ is a po- $n$-system.

There exists $c \in \mathrm{S} \backslash \mathrm{Q}$ such that $c \leq a \alpha x \beta a$ for some $x \in \mathrm{S}, \alpha, \beta \in \Gamma$.

$c \leq a \alpha x \beta a \in a \Gamma \mathrm{S}^{1} \Gamma a \subseteq \mathrm{Q}$. Thus $c \in \mathrm{Q}$.

It is a contradiction. Therefore $a \in \mathrm{Q}$. Hence $\mathrm{Q}$ is a semiprime po- $\Gamma$-ideal of $\mathrm{S}$.

THEOREM $5.17:$ If $\mathrm{N}$ is an $n$-system in a po- $\Gamma$-semigroup $S$ and $a \in \mathrm{N}$, then there exists an $m$-system $M$ in $S$ such that $a \in M$ and $M \subseteq N$.

Proof: We construct a subset M of $\mathrm{N}$ as follows. Define $a_{l}=a$.

Since $a_{l} \in \mathrm{N}$ and $\mathrm{N}$ is an $n$-system, there exists $c_{1} \in \mathrm{N}$ such that $c_{1} \leq a_{1} \alpha x \beta a_{1}$ for some $x \in \mathrm{S}, \alpha, \beta \in \Gamma$. Then $c_{1} \in\left(a_{l} \Gamma \mathrm{S} \Gamma a_{l}\right]$. Thus $\left(a_{l} \Gamma \mathrm{S} \Gamma a_{1}\right] \cap \mathrm{N} \neq \emptyset$. Let $a_{2} \in\left(a_{l} \Gamma \mathrm{S} \Gamma a_{l}\right] \cap \mathrm{N}$.

Since $a_{2} \in \mathrm{N}$ and $\mathrm{N}$ is an $n$-system, there exists $c_{2} \in \mathrm{N}$ such that $c_{2} \leq a_{2} \alpha x \beta a_{2}$ for some $x \in \mathrm{S}, \alpha, \beta \in \Gamma$. Then $c_{2} \in\left(a_{2} \Gamma \mathrm{S} \Gamma a_{2}\right]$. Thus $\left(a_{2} \Gamma \mathrm{S} \Gamma a_{2}\right] \cap \mathrm{N} \neq \varnothing$ and so on.

In general, if $a_{i}$ has been defined with $a_{i} \in \mathrm{N}$, choose $a_{i+1}$ as an element of $\left(a_{i} \Gamma \mathrm{S} \Gamma a_{i}\right] \cap \mathrm{N}$ there exists $c_{\mathrm{i}+1} \in \mathrm{N}$ such that $c_{i+1} \leq a_{i+1} \alpha x \beta a_{i+1}$ for some $x \in \mathrm{S}, \alpha, \beta \in \Gamma$.

Then $c_{\mathrm{i}+1} \in\left(a_{\mathrm{i}+1} \Gamma \mathrm{S} \Gamma a_{\mathrm{i}+1}\right]$. Thus $\left(a_{i+1} \Gamma \mathrm{S} \Gamma a_{i+1}\right] \cap \mathrm{N} \neq \emptyset$.

Let $\mathrm{M}=\left\{a_{1}, a_{2}, \ldots \ldots, a_{i}, a_{i+1} \ldots \ldots\right\}$. Now $a \in \mathrm{M}$ and $\mathrm{M} \subseteq \mathrm{N}$.

We now show that $\mathrm{M}$ is an $m$-system.

Let $a_{i}, a_{j} \in \mathrm{M}$. If $i=j$ then, for the element $a_{j+1} \in \mathrm{S}$, We have $a_{i+1} \in\left(a_{i} \Gamma \mathrm{S} \Gamma a_{i}\right] \subseteq\left(a_{i} \Gamma \mathrm{S} \Gamma a_{j}\right]$

$\Rightarrow a_{i+1} \leq a_{i} \alpha x \beta a_{j}, x \in \mathrm{S}, \alpha, \beta \in \Gamma$.

If $i<j$ then, for the element $a_{j+1} \in \mathrm{S}$,

We have $a_{j+1} \in\left(a_{i} \Gamma \mathrm{S} \Gamma a_{i}\right] \subseteq\left(\left(a_{\mathrm{j}-1} \Gamma \mathrm{S} \Gamma a_{\mathrm{j}-1}\right] \mathrm{S} a_{\mathrm{j}}\right] \subseteq\left(a_{\mathrm{j}-1} \Gamma \mathrm{S} \Gamma a_{\mathrm{j}}\right] \subseteq \ldots \subseteq\left(a_{\mathrm{i}} \Gamma \mathrm{S} \Gamma a_{\mathrm{j}}\right]$.

Hence $a_{j+1} \leq a_{i} \alpha x \beta a_{j} \in \mathrm{S}$, for $x \in \mathrm{S} \alpha, \beta \in \Gamma$.

If $j<i$ then, for the element $a_{i+1} \in \mathrm{S}$.

We have $a_{i+1} \in\left(a_{i} \Gamma \mathrm{S} \Gamma a_{i}\right] \subseteq\left(a_{\mathrm{i}} \Gamma \mathrm{S} \Gamma\left(a_{\mathrm{i}-1} \mathrm{~S} a_{\mathrm{i}-1}\right)\right] \subseteq\left(a_{\mathrm{i}} \Gamma \mathrm{S} \Gamma a_{\mathrm{i}-1}\right] \subseteq \ldots \subseteq\left(a_{\mathrm{i}} \Gamma \mathrm{S} \Gamma a_{\mathrm{j}}\right]$

Therefore $\mathrm{M}$ is an $m$-system.

\section{VI . CONCLUSION}

It is proved that (1) every completely semiprime po- $\Gamma$-ideal of a po- $\Gamma$-semigroup is a semiprime po- $\Gamma$-ideal, (2) every po- completely prime $\Gamma$-ideal of a po- $\Gamma$-semigroup is a po-completely semiprime $\Gamma$-ideal. It is also proved that the nonempty intersection of any family of (1)a po- completely prime $\Gamma$-ideals of a po- $\Gamma$-semigroup is a po- completely semiprime $\Gamma$-ideal, (2)a po- prime $\Gamma$-ideals of a po- $\Gamma$-semigroup is a semiprime po- $\Gamma$-ideal. It is also proved that a po- $\Gamma$-ideal $\mathrm{Q}$ of a po- $\Gamma$-semigroup $\mathrm{S}$ is a semiprime iff $\mathrm{S} \backslash \mathrm{Q}$ is either an $\mathrm{n}$-system or empty. Further it is proved that if $\mathrm{N}$ is an $\mathrm{n}$-system in a po- $\Gamma$-semigroup $\mathrm{S}$ and a $\in \mathrm{N}$, then there exists an $\mathrm{m}$ - 
system $\mathrm{M}$ of $\mathrm{S}$ such that $\mathrm{a} \in \mathrm{M}$ and $\mathrm{M} \subseteq \mathrm{N}$. The study of ideals in semigroups, $\Gamma$-semigroups creates a platform for the ideals in po- $\Gamma$-semigroups.

\section{VII . Acknowledgements}

I thank the Management, Principal and the staff of VKR, VNB \& AGK College of Engineering for giving me their valuable support in preparing this paper.

\section{REFERENCES}

[1] Anjaneyulu. A, and Ramakotaiah. D., On a class of semigroups, Simon stevin, Vol.54(1980), 241-249.

[2] Anjaneyulu. A., Structure and ideal theory of Duo semigroups, Semigroup Forum, Vol.22(1981), 257-276.

[3] Anjaneyulu. A., Semigroup in which Prime Ideals are maximal, Semigroup Forum, Vol.22(1981), 151-158.

[4] Clifford. A.H. and Preston. G.B., The algebraic theory of semigroups, Vol-I, American Math.Society, Providence(1961)

[5] Clifford. A.H. and Preston. G.B., The algebraic theory of semigroups, Vol-II, American Math.Society, Providence(1967)

[6] Chinram. R and Jirojkul. C., On bi- $\Gamma$-ideal in $\Gamma$ - Semigroups, Songklanakarin J. Sci. Tech no.29(2007), 231-234.

[7] Giri. R. D. and Wazalwar. A. K., Prime ideals and prime radicals in non- commutative semigroup, Kyungpook Mathematical Journal Vol.33(1993), no.1, 37-48.

[8] Dheena. P. and Elavarasan. B., Right chain a po-Г-semigroups, Bulletin of the Institute of Mathematics Academia Sinica (New Series) Vol. 3 (2008), No. 3, pp. 407-415

[9] Kostaq Hila., Filters in a po- $\Gamma$-semigroups, Rocky Mountain Journal of Mathematics Volume 41, Number 1, 2011.

[10] Kwon. Y. I. and Lee. S. K., Some special elements ina po- Г-semigroups, Kyungpook Mathematical Journal., 35 (1996), $679-685$.

[11] Madhusudhana Rao. D, Anjaneyulu. A and Gangadhara Rao. A, Pseudo symmetric $\Gamma$-ideals in $\Gamma$-semigroups, International eJournal of Mathematics and Engineering 116(2011) 1074-1081.

[12] Madhusudhana rao. D, Anjaneyulu. A \& Gangadhara rao. A, Prime $\Gamma$-radicals in $\Gamma$-semigroups, International eJournal of Mathematics and Engineering 138(2011) 1250 - 1259.

[13] Manoj Siripitukdet and Aiyared Iampan, On the ordered n-prime ideals in ordered $\Gamma$-semigroups, Commun. Korean Math. Sco. 23 (2008), No. 1, pp. 19-27.

[14] Niovi Kehayopulu., m-systems and n-systems ina po-semigroups, Quasigroups and Related systems 11(2004), 55-58.

[15] Petrch. M., Introduction to semigroups, Merril Publishing Company, Columbus, Ohio,(973).

[16] Ronnason Chinram and Kittisak Tinpun., A Note on Minimal Bi-Ideals ina po- $\Gamma$-semigroups, International Mathematical Forum, 4 , 2009, no. 1, 1-5.

[17] Samit Kumar Manjumder and Sujit Kumar Sardar., On properties of fuzzy ideals in a po-semigroups, Armenian Journal of Mathematics, Volume 2, Number 2, 2009, 65-72.

[18] Sen. M.K. and Saha. N.K., On $\Gamma$ - Semigroups-I, Bull. Calcutta Math. Soc. 78(1986), No.3, 180-186.

[19] Sen. M.K. and Saha. N.K., On Г - Semigroups-II, Bull. Calcutta Math. Soc. 79(1987), No.6, 331-335

[20] Sen. M.K. and Saha. N.K., On Г - Semigroups-III, Bull. Calcutta Math. Soc. 8o(1988), No.1, 1-12. 